\title{
SOME DISCRETE PROPERTIES OF THE SPACE OF LINE TRANSVERSALS TO DISJOINT BALLS
}

\author{
XAVIER GOAOC
}

\begin{abstract}
Attempts to generalize Helly's theorem to sets of lines intersecting convex sets led to a series of results relating the geometry of a family of sets in $\mathbb{R}^{d}$ to the structure of the space of lines intersecting all of its members. We review recent progress in the special case of disjoint Euclidean balls in $\mathbb{R}^{d}$, more precisely the inter-related notions of cone of directions, geometric permutations and Helly-type theorems, and discuss some algorithmic applications.
\end{abstract}

Key words. Geometric transversal, Helly's theorem, line, sphere, geometric permutation, cone of directions.

1. Introduction. Lines intersecting or tangent to prescribed geometric objects are central to various problems in computational geometry and application areas; typical examples include visibility [26, 64] or shortest path [61] computation and robust statistics $[14,67]$. To design efficient algorithms for these problems, one first has to understand the geometry of the underlying sets of lines. A natural embedding of the space of lines in $\mathbb{P}^{3}(\mathbb{R})$ is as a quadric in $\mathbb{P}^{5}(\mathbb{R})$, the Klein (or Plücker) quadric; in some sense this is optimal ${ }^{1}$, so line geometry is, at least in dimension 3 , inherently nonlinear.

Let $\mathcal{C}$ be a collection of subsets of $\mathbb{R}^{d}$, or objects for short. Denote by $\mathcal{T}_{k}(\mathcal{C})$ the set of $k$-transversals to $\mathcal{C}$, that is of $k$-dimensional affine subspaces that intersect every member of $\mathcal{C}$. Helly's theorem [42] asserts that if $\mathcal{C}$ consists of convex sets then $\mathcal{T}_{0}(\mathcal{C})$ is nonempty if and only if $\mathcal{T}_{0}(F)$ is nonempty for any subset $F \subset \mathcal{C}$ of size at most $d+1$. Whether Helly's theorem generalizes to other values of $k$ is a natural question which was, to my knowledge, first investigated in the 1930's by Vincensini [76]. The answer turns out to be negative in general but positive when the geometry of the objects is adequately constrained. The study of how the geometry of the objects in $\mathcal{C}$ determines the structure of $\mathcal{T}_{k}(\mathcal{C})$, and subsequent developments of similar flavor, is now designated as geometric transversal theory [34].

Helly's theorem was recently generalized to line transversals $(k=1)$ to disjoint (Euclidean) balls in $\mathbb{R}^{d}$, answering in the positive a conjecture of Danzer [23] who settled the 2-dimensional case. This generalization builds on a series of results concerning two notions: cone of directions and geometric permutations. This survey gives a comprehensive overview of these investigations by presenting, in a unified language, the results of several papers $[3,7,16,17,19,20,21$, $45,48,53,73,81]$, new extensions of these results and some of their algorithmic consequences. Although some results generalize to other settings, the discussion will focus on the case of line transversals to disjoint balls.

\footnotetext{
${ }^{1}$ Indeed (i) there does not exist any homeomorphism between the lines in $\mathbb{R}^{3}$ and an open subset of $\mathbb{P}^{4}(\mathbb{R})$, and (ii) any algebraic homeomorphism between lines in $\mathbb{R}^{3}$ and points in $\mathbb{P}^{5}(\mathbb{R})$ has degree at least 2 [65, Remarks 2.1.4 and 2.1.6, p. 143].
} 
1.1. Notations and terminology. We denote by $\mathbb{R}^{d}$ the real $d$-dimensional affine space or, equivalently, the Euclidean $d$-dimensional space; the Euclidean metric is the only one we consider over $\mathbb{R}^{d}$. We denote by $\mathbb{P}^{d}$ the real $d$-dimensional projective space and by $\mathbb{S}^{d-1}$ the space of directions in $\mathbb{R}^{d}$, which we identify with the unit sphere. Recall that a great circle of $\mathbb{S}^{d}$ is a section of $\mathbb{S}^{d}$ by some 2 -flat through its center. We write $A^{o}$ the interior of a set $A$ and use arrows to denote vectors; in particular, we write $\vec{\ell}$ a direction vector of an oriented line $\ell$. We use $\langle\vec{u}, \vec{v}\rangle$ and $\angle(\vec{u}, \vec{v})$ to denote, respectively, the dot product of and the angle between vectors $\vec{u}$ and $\vec{v}$.

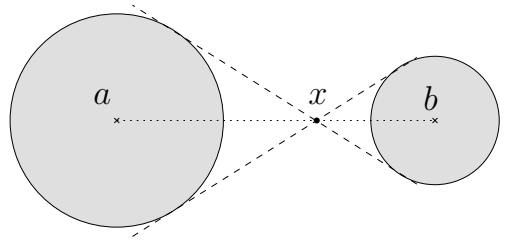

FIG. 1. The internal center of similitude $x$ of two balls, represented in a 2-plane through their centers.

A ball is closed unless otherwise specified: the ball of center $c$ and radius $r$ is the set of points $x$ such that $|c-x| \leq r$. In particular, disjoint balls are not allowed to be tangent; for the sake of simplicity, we say that several balls are disjoint if they are pairwise disjoint. A unit ball is a ball with radius 1 ; since transversal properties are unchanged under scaling, results obtained for unit balls usually extend to congruent balls, i.e. sets of balls with equal radii. The radius disparity of a set of balls is the ratio of the largest radius to the smallest. The internal center of similitude of two disjoint balls in $\mathbb{R}^{d}$ with respective centers $a, b$ and radii $r_{a}, r_{b}$ is the point $\frac{r_{b} a+r_{a} b}{r_{a}+r_{b}}$ (see Figure 1); this point is sometimes referred to as the geometric center [81] or the center of gravity [47] of the two balls.

We use the terms collection or family for an unordered set, and sequence for an ordered set. We denote by $|X|$ the cardinality of a set $X$. Given a sequence $\mathcal{C}$, we denote by $\prec_{\mathcal{C}}$ the corresponding ordering on its elements. A subsequence of a sequence is a subset of its members, ordered as in the sequence. A $k$-transversal to a collection $\mathcal{C}$ is an affine subspace of dimension $k$ that intersects every member of $\mathcal{C}$; for the sake of simplicity, we say transversal for 1-transversal, that is line transversal, and speak of the common intersection of a family for the common intersection of its members. Depending on the context, a

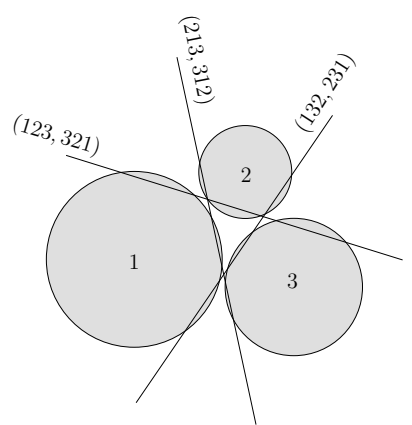

FIG. 2. Three disks with three geometric permutations. transversal may be oriented or not. An oriented transversal to a collection $\mathcal{C}$ of convex sets induces an ordering on $\mathcal{C}$, and a partial ordering if some of its members intersect. A geometric permutation of $\mathcal{C}$ is a pair of orderings, one reverse of the other, on $\mathcal{C}$ induced by the two orientations of some transversal (see Figure 2). A transversal to a sequence $\mathcal{C}$ is order-respecting if it meets the members of $\mathcal{C}$ in the order $\prec_{\mathcal{C}}$. The directions of all order-respecting 
transversals to $\mathcal{C}$ make up a subset of $\mathbb{S}^{d-1}$ called the cone of directions of $\mathcal{C}$ and denoted $\mathcal{K}(\mathcal{C})$.

The projection along a direction $\vec{u}$ is the orthogonal projection on some hyperplane with normal $\vec{u}$; since we consider properties invariant under translation of the hyperplane, all such hyperplanes are equivalent.

A pinning configuration is a ordered pair $(\mathcal{C}, \ell)$ where $\mathcal{C}$ is a collection of objects having $\ell$ as an isolated transversal, in the sense that $\ell$ is an isolated point of $\mathcal{T}_{1}(\mathcal{C})$. Equivalently we say that $\mathcal{C}$ pins the line $\ell$. A pinning configuration $(\mathcal{C}, \ell)$ is minimal if no proper subset of $\mathcal{C}$ pins $\ell$.

1.2. Content and organization. We start by recalling, in Section 2 , some variants of Helly's theorem used in the rest of the paper. We then discuss, in Section 3, the convexity of the connected components of the projection of $\mathcal{T}_{1}(\mathcal{C})$ in the space of directions, the so-called cones of directions, when $\mathcal{C}$ consists of disjoint balls; we present the two existing approaches to proving this result [3, 16, $19,40,45]$ and discuss some of its immediate consequences. In Section 4, we review the bounds obtained on the number of geometric permutations of disjoint balls [7, 20, 21, 48, 53, 73, 81]. Section 5 then discusses Helly-type theorems for line transversals to disjoint balls. Specifically, we present the known bounds on the constants $k$ for which the following statements hold:

(a) a sequence of disjoint balls has a transversal if every subsequence of size at most $k$ has an order-respecting transversal,

(b) a collection of disjoint balls (with adequate constraints on the radii) has a transversal if every subset of size at most $k$ has a transversal,

(c) if a transversal to $n$ disjoint balls is isolated then it is an isolated transversal to a subset of at most $k$ of the balls.

The smallest such constants are referred to as, respectively, the Hadwiger, Helly ${ }^{2}$ and pinning numbers. Section 6 reviews the connection between Helly-type theorems and LP-type problems [4] and the impact of the bounds on the Helly number on the computational complexity of finding a transversal to a family of disjoint balls. We conclude this paper by commenting some open problems in Section 7.

1.3. Related surveys. For an overview of geometric transversal theory we refer to the surveys of Wenger [79] and Goodman et al. [35]. A detailed account on early generalizations of Helly's theorem can be found in the article by Danzer et al. [24] and more recent developments are presented in the survey of Eckhoff [27]. The overlap of the present survey has with the, related, ones of Sottile and Theobald [74] and Holmsen [44] is limited, so they can be read in conjunction. For a discussion of the computational aspects of lines in space we refer to the survey of Pellegrini [64] and the notes of the Alcala lecture by Pach and Sharir [63, Chapter 7].

2. Helly's theorem. Helly's theorem [42] of $1923{ }^{3}$ forms, together with Radon's and Caratheodory's theorems, the basis of convex geometry [57].

\footnotetext{
${ }^{2}$ That is, the Helly number for sets of line transversals in the classical sense.

${ }^{3}$ It is sometimes dated from 1913, the year when Helly communicated it to Radon [24].
} 
THEOREM 2.1. A finite family of $n \geq d+1$ convex sets in $\mathbb{R}^{d}$ has a point in common if and only if every $d+1$ members have a point in common.

One way to restate Helly's theorem is that the emptiness of the intersection of any finite number of convex sets in $\mathbb{R}^{d}$ can be decided by looking only at subsets of size at most $d+1$. Other results of similar flavor are called Helly-type theorems; the typical formulation is that a collection $\mathcal{C}$ has property $\mathcal{P}$ if and only if every subset of $\mathcal{C}$ of size at most $k$ has property $\mathcal{P}$ ( $k$ being independent of $|\mathcal{C}|$ ). The smallest integer $k$ for which a given theorem holds is called the associated Helly number. In this section, we review some of these results used when dealing with transversals.

2.1. Spherical Helly theorem. There are several generalizations of Helly's theorem on the sphere $\mathbb{S}^{d}$ involving various notions of convexity [66]. Recall that a set $A \subset \mathbb{S}^{d}$ is strongly convex if it does not contain any pair of antipodal points and if it contains for any two points in $A$ the smallest great circle arc that connects them. A strongly convex set $A \subset \mathbb{S}^{d}$ is strictly strongly convex if any great circle intersects its boundary in at most two points.

THEOREM 2.2. A finite family of $n \geq d+2$ strongly convex sets in $\mathbb{S}^{d}$ has a point in common if and only if every $d+2$ members have a point in common.

Proof. Consider a family $\mathcal{C}$ of strongly convex sets on a sphere $\mathbb{S}^{d}$ embedded in $\mathbb{R}^{d+1}$ with center $O$. Replacing each set $X \in \mathcal{C}$ by $X^{\prime}=C H(X \cup\{O\}) \backslash\{O\}$, where $C H(\cdot)$ denotes the convex hull operator, we get a family $\mathcal{C}^{\prime}$ of convex sets in $\mathbb{R}^{d+1}$ that has a common intersection if and only if $\mathcal{C}$ has a common intersection. The statement follows.

With additional constraints on the sets one may reduce the Helly number to $d+1$. One simple example is if all sets in the family are contained in some open hemisphere of $\mathbb{S}^{d}$, as one can map that hemisphere to $\mathbb{R}^{d}$ while preserving the convexity structure. Another situation of interest is when the diameter ${ }^{4}$ of the sets is bounded [66, Theorem 3]:

THEOREM 2.3. A finite family of $n \geq d+2$ convex sets in $\mathbb{S}^{d}$, each of diameter less than $\frac{2 \pi}{3}$, has nonempty intersection if and only if every $d+1$ members have nonempty intersection.

2.2. Topological Helly theorem. Helly's theorem still holds if convexity is replaced by some weaker topological condition. Recall that a homology cell is a nonempty set with trivial homology. In particular, we use the following variant [25] of Helly's topological theorem [43]:

THEOREM 2.4. Let $\mathcal{C}$ be a finite family of open subsets of $\mathbb{R}^{d}$ such that the intersection of any $r$ elements of $\mathcal{C}$ is a homology cell for $r \leq d$. Then all sets in $\mathcal{C}$ have a point in common if and only if every $d+1$ members $d o$.

Recall that a set is contractible if it is homotopic to a point. The above theorem remains true if "homology cell" is replaced by "contractible set" since contractible subsets of $\mathbb{R}^{d}$ are homology cells (homology being invariant under homotopy); we will, in fact, only need this simpler variant.

\footnotetext{
${ }^{4}$ The diameter of $X \subset \mathbb{S}^{d}$ is the maximal opening angle of any great circle arc contained in $X$.
} 
2.3. Helly's theorem for unions of sets. The previous theorems do not apply to families of disconnected sets, as they are neither convex nor homologically trivial. Helly's theorem does, however, generalize to collections such that any members intersect in a bounded number of convex sets. The following theorem was conjectured by Grünbaum and Motzkin [39] and proven by Amenta [5]:

THEOREM 2.5. Let $\mathcal{C}$ be a collection of sets in $\mathbb{R}^{d}$ such that the intersection of any nonempty finite sub-family of $\mathcal{C}$ is the disjoint union of at most $k$ closed convex sets. Then all sets in $\mathcal{C}$ have a point in common if and only if every $k(d+1)$ members do.

The same argument as in the proof of Theorem 2.2 yields:

COROLLARY 2.1. Let $\mathcal{C}$ be a collection of sets in $\mathbb{S}^{d}$ such that the intersection of any nonempty finite sub-family of $\mathcal{C}$ is the disjoint union of at most $k$ closed convex sets. Then all sets in $\mathcal{C}$ have a point in common if and only if every $k(d+2)$ members do.

Similar generalizations were obtained for the topological versions of Helly's theorem $[2,58]$. We use the following corollary of a theorem of Matoušek [58, Theorem 2]:

THEOREM 2.6. For any $d \geq 2, k \geq 1$ there exists a number $h(d, k)$ such that the following holds. Let $\mathcal{C}$ be a collection of sets in $\mathbb{R}^{d}$ such that the intersection of any nonempty finite sub-family of $\mathcal{C}$ has at most $k$ path-connected components, each of them contractible. Then $\mathcal{C}$ has a point in common if and only if every $h(k, d)$ members have a point in common.

2.4. Convexity structure on the Grassmannian. Transversals to convex sets provide an elegant way to define a "convexity" structure on the Grassmaniann [31,33]: convex sets of $k$-flats are simply defined as the sets of $k$-transversals to convex objects. When $k=1$ and the objects are restricted to axis-aligned boxes, the resulting structure is known as frame convexity. In fact, frame convexity, when restricted to ascending lines ${ }^{5}$, is isomorphic to the ordinary notion of convexity on some convex subset of $\mathbb{R}^{2 d-2}$; through this isomorphism, Helly's theorem essentially corresponds to Santaló's theorem [68], one of the earliest Helly-type theorems for line transversals [32]. As the examples of Santaló [68] and Danzer [23] show, Helly's theorem does not extend to the more general convexity structure of Goodman and Pollack [33].

3. Cone of directions. One of the specificities of the set of transversals to disjoint balls ${ }^{6}$ is the following convexity property [16, Theorem 1]:

THEOREM 3.1. The cone of directions of any sequence of disjoint balls in $\mathbb{R}^{d}$ is a strictly, strongly convex subset of $\mathbb{S}^{d-1}$.

The use of the convexity of the cone of directions for proving Helly-type theorems for line transversals can be traced back to Vincensini [76]. In dimension 3

\footnotetext{
${ }^{5} \mathrm{~A}$ line is ascending with respect to a coordinate frame if it can be oriented so that all coordinates are nondecreasing.

${ }^{6}$ This property implies that the Hadwiger number is bounded (see Lemma 5.1), which is not the case for disjoint translates of a convex set [46].
} 
or more, Theorem 3.1 was first asserted ${ }^{7}$ for the case of thinly distributed families of balls [40], i.e. families where the distance between the centers of any two balls is at least twice the sum of their radii.

3.1. Reduction. We first explain why Theorem 3.1 follows from the case of 3 balls in 3 dimensions.

LEMMA 3.1. If $\mathcal{C}$ is a sequence of disjoint balls in $\mathbb{R}^{d}$ then $\mathcal{K}(\mathcal{C})$ is convex if $\mathcal{K}(X \cap T)$ is convex for any triple $X \subset \mathcal{C}$ and any 3-transversal $T$ to $X$.

Proof. The statement follows from two facts: (i) $\mathcal{K}(\mathcal{C})$ is convex if $\mathcal{K}(\mathcal{C} \cap T)$ is convex for every 3 -transversal $T$ of $\mathcal{C}$ and (ii) $\mathcal{K}(\mathcal{C})$ is convex if $\mathcal{K}(X)$ is convex for any subsequence $X \subset \mathcal{C}$ of size $d$.

Let $\ell$ and $\ell^{\prime}$ be two order-respecting transversals to a sequence $\mathcal{C}$ of disjoint convex sets in $\mathbb{R}^{d}$ and $T$ some $^{8} 3$-space that contains their span. Observe that $T$ is a 3 -transversal to $\mathcal{C}$ whose vector space contains any direction in the great circle spanned by $\vec{\ell}$ and $\overrightarrow{\ell^{\prime}}$. Thus, if $\mathcal{K}(\mathcal{C} \cap T)$ is convex then $\mathcal{K}(\mathcal{C})$ contains the shorter arc of the great circle between $\vec{\ell}$ and $\overrightarrow{\ell^{\prime}}$. As a consequence, we get that if $\mathcal{K}(\mathcal{C} \cap T)$ is convex for every 3 -transversal to $\mathcal{C}$, then $\mathcal{K}(\mathcal{C})$ is convex. This proves claim (i).

Applying Helly's theorem to the projection of $\mathcal{C}$ along some direction $\vec{u}$, we find that $\mathcal{C}$ has a transversal with direction $\vec{u}$ if and only if every subset of size $d$ has a transversal with direction $\vec{u}$. Since two parallel lines intersect disjoint convex sets in the same order,

$$
\mathcal{K}(\mathcal{C})=\bigcap_{X \subset \mathcal{C},|X|=d} \mathcal{K}(X)
$$

and claim (ii) follows.

This reduction holds more generally for sequences of disjoint convex sets.

3.2. Analytic approach. The first published proof of Theorem 3.1 came for families of disjoint unit balls in $\mathbb{R}^{3}$ [45]. This case was not covered by Hadwiger's statement since two unit balls within distance $\delta \in(0,2)$ are disjoint but not thinly distributed.

Let $\mathcal{C}$ be a sequence of disjoint unit balls in $\mathbb{R}^{3}$ and $u, v \in \mathcal{K}(\mathcal{C})$. Consider some coordinate axis, say $z$, normal to these two directions and let $R$ denote some plane parallel to $z$. Let $Q_{\mathcal{C}}^{u v} \subset \mathbb{R} \times \mathbb{S}^{1}$ denote the set of $(t, \alpha)$ such that there is an oriented line in the plane $z=t$ that intersects the sequence $\mathcal{C}$ in the right order and makes an angle $\alpha$ with $R$. The projection of $Q_{\mathcal{C}}^{u v}$ on the second coordinate is exactly the intersection of $\mathcal{K}(\mathcal{C})$ with the great circle through $u$ and $v$; the convexity of $Q_{\mathcal{C}}^{u v}$ for all pairs $u, v \in \mathcal{K}(\mathcal{C})$ thus implies that of $\mathcal{K}(\mathcal{C})$. Let $A$ and $B$ be two balls such that $A \prec B$ in $\mathcal{C}$; define similarly $Q_{A B}^{u v} \subset \mathbb{R} \times \mathbb{S}^{1}$ as the set of all $(t, \alpha)$ such that there is an oriented line in the plane $z=t$ that intersects

\footnotetext{
${ }^{7}$ Although Hadwiger's article does not contain a proof of this claim, its editor seems to have been provided with the details [3].

${ }^{8}$ If $\ell$ and $\ell^{\prime}$ are skew $T$ is unique.
} 
$A$ before $B$ and makes an angle $\alpha$ with $R$. Helly's theorem implies that

$$
Q_{\mathcal{C}}^{u v}=\bigcap_{A \prec B \text { in } \mathcal{C}} Q_{A B}^{u v}
$$

so it suffices to prove the convexity of $Q_{A B}^{u v}$. Using symmetries with respect to translation and rotation, the convexity of $Q_{A B}^{u v}$ reduces to the convexity of the function

$$
G: t \mapsto \arcsin \left(\frac{\sqrt{1-z^{2}}+\sqrt{1-(z-b)^{2}}}{a}\right),
$$

on the interval $\left[\frac{b}{2}, 1\right]$, where $a$ and $b$ parameterize the respective positions of the centers. Elementary calculus suffices to conclude.

3.3. Extending the analytic approach. The convexity of $Q_{A B}^{u v}$ is stronger than that of the cone of directions: it requires that if $\mathcal{C}$ has transversals with directions $\vec{u}$ and $\vec{v}$ in planes $z=z_{u}$ and $z=z_{v}$, then for any $t \in[0,1]$ it has a transversal with direction $t \vec{u}+(1-t) \vec{v}$ in the plane $z=t z_{u}+(1-t) z_{v}$. This property does, in fact, not hold for disjoint balls with arbitrary radii; Figure 3 depicts an example of two 3-dimensional disjoint balls for which the nonconvexity of certain sets $Q_{\mathcal{C}}^{u v}$ can be ascertained [36]; the balls have centers $(0,0,0)$ and $(3.9,0,8.6)$ and radii 1 and 8.44. Of course, this nonconvexity may (and, in fact, does) disappear when $Q_{\mathcal{C}}^{u v}$ is projected on the second coordinate, so this does not disprove Theorem 3.1. It does, however, show that the previous approach requires a constraint stronger than the balls' disjointedness. That approach was, nevertheless, extended in two directions.

Ambrus et al. [3] used this technique to prove Theorem 3.1 for $d$-dimensional unit balls such that the distance between any two centers is at least $2 \sqrt{2+\sqrt{2}}$. The key observation is that the distance between two centers in the section of such a sequence by any of its 3 -transversals is at least $2 \sqrt{1+\sqrt{2}}$; this guarantees the convexity of the function $G$ for these sections, and thus for the $d$-dimensional balls (by the argument used to prove Lemma 3.1).

Cheong et al. [19] proved Theorem 3.1 for sequences of balls where every pair is isometric to the section of two higher-dimensional disjoint unit balls by some $d$-transversal; such pairwise-inflatable pairs are characterized by the property that the squared distance between their centers is at least twice the sum of their squared radii ${ }^{9}$. The convexity of $Q_{A B}^{u v}$ is first established for disjoint unit balls in $\mathbb{R}^{4}$ via extensive computations ${ }^{10}$, then deduced for pairwise-inflatable balls in $\mathbb{R}^{3}$ and finally extended to pairwise-inflatable balls in $\mathbb{R}^{d}$.

\footnotetext{
${ }^{9}$ This is, somewhat unexpectedly, simply Hadwiger's condition of being thinly-distributed where every distance is replaced by its square. Note that disjoint unit balls are pairwise-inflatable.

${ }^{10}$ Although computer algebra systems such as Maple [56] were instrumental in developing these computations, the resulting proof can still be checked manually.
} 

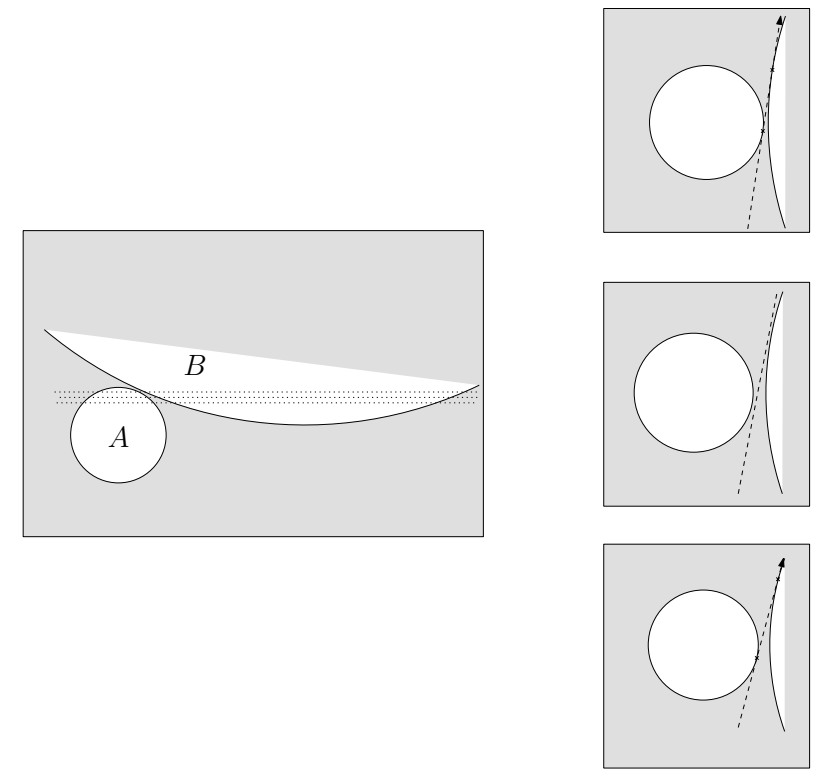

FIG. 3. Two balls such that $Q_{A B}^{u v}$ is not convex, in the $(x, z)$ plane (left). Three slices at $(x, y)$ planes (regularly spaced along the $y$ axis) showing that the "middle" of two existing transversals is not a transversal (right).

3.4. The algebraic approach. The general case of Theorem 3.1 was proven by Borcea et al.[16] by showing that the algebraic arcs that make up the boundary of $\mathcal{K}(\mathcal{C})$ do not contain any inflexion point. By Lemma 3.1, it suffices to consider the case where $\mathcal{C}$ is a triple of disjoint balls in $\mathbb{R}^{3}$.

Boundary arcs. The directions that belong to the boundary of $\mathcal{K}(\mathcal{C})$ can be characterized in terms of projection patterns [19, Lemma 9 and 11]:

LEMMA 3.2. A direction $\vec{u}$ belongs to the boundary of $\mathcal{K}(\mathcal{C})$ if and only if the projections of the balls of $\mathcal{C}$ along $\vec{u}$ intersect in a single point.

An immediate consequence is that the directions in the interior of $\mathcal{K}(\mathcal{C})$ are exactly the directions of transversals to the open balls in $\mathcal{C}$ :

$$
\mathcal{K}\left(\mathcal{C}^{o}\right)=\mathcal{K}^{o}(\mathcal{C}) .
$$

The intersection of the projections of a triple $\mathcal{C}$ of balls in $\mathbb{R}^{3}$ along $\vec{u} \in \partial \mathcal{K}(\mathcal{C})$ belongs to the boundary of either two or three disks (see Figure 4). The boundary of $\mathcal{K}(\mathcal{C})$ thus decomposes into two types of arcs, directions of inner special bitangents, i.e. tangents to two balls through their inner center of similitude ${ }^{11}$, and of tritangents. The directions of inner special bitangents make up a circle on $\mathbb{S}^{2}$, so the local convexity of arcs of the first type is trivial. The directions of tritangents

\footnotetext{
${ }^{11}$ These lines are exactly the tangents to two balls contained in a common tangent plane; they are sometimes referred to as limiting bitangents.
} 

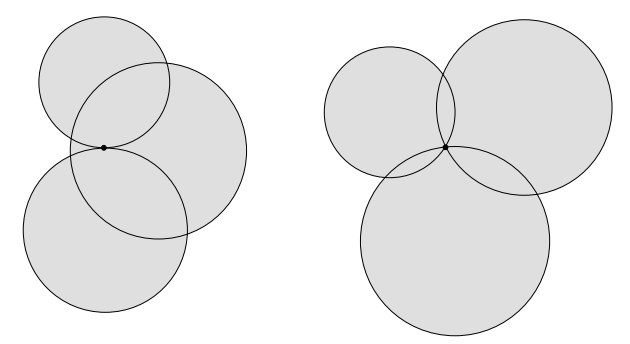

FIG. 4. Intersection patterns for the projections of three balls along a direction on the boundary of their cone of directions: direction of inner special bitangent (left) and of tritangent(right).

make up an algebraic curve of degree 6 on $\mathbb{S}^{2}$, the direction-sextic of the triple of balls, that is not convex in general. It is thus important to identify the directions of tritangents that belong to the boundary of $\mathcal{K}(\mathcal{C})$ [16, Proposition 3]:

LEMMA 3.3. The direction of a tritangent $\ell$ is on the boundary of the cone of directions of three balls (for the adequate ordering) if and only if $\ell$ intersects the triangle spanned by their centers.

This generalizes to higher dimensions and follows from the property that the balls centered at the vertices of a simplex and going through a given point have no other common intersection if and only if that point belongs to the simplex.

Controlling the flexes. Proving Theorem 3.1 essentially amounts to showing that the boundary of the cone of directions of three disjoint balls does not contain inflexion points of the curve of directions of tritangents; these, also called flexes, are the intersections of the curve with its Hessian and the sources of nonconvexity in an algebraic curve. Given a projection pattern of a sequence $\mathcal{C}$ of three balls along some direction $\vec{u} \in \partial \mathcal{K}(\mathcal{C})$, the conditions that the Hessian of the direction-sextic of $\mathcal{C}$ vanishes in $\vec{u}$, and that the balls are disjoint exclude one another [16, Proposition 5]. This approach avoids the apparently difficult task of classifying the 72 flexes of the direction-sextic ${ }^{12}$.

3.5. Strict convexity and tangents to spheres. The algebraic approach immediately yields that the cone of direction is strictly convex, in the sense that its boundary does not contain great circle arcs. This property is also related [19, Proposition 4] to collections of spheres with degenerate families of common tangents [74]. In $\mathbb{R}^{3}$, if the cone of directions of three balls contains a great circle arc then these balls have infinitely many common tangents that meet one and the same line at infinity [19, Lemma 10]. Such configurations require the balls to intersect [60], so the strict convexity follows for three, and hence $n$, disjoint balls in $\mathbb{R}^{3}$. The generalization to higher dimensions is based on the following lemma:

LEMMA 3.4. For any sequence $\mathcal{C}$ of disjoint balls in $\mathbb{R}^{d}$ and any great circle $\Gamma \subset \mathbb{S}^{d-1}$ there exists a 3 -transversal $T$ to $\mathcal{C}$ such that $\mathcal{K}(\mathcal{C}) \cap \Gamma=\mathcal{K}(\mathcal{C} \cap T) \cap \Gamma$. Moreover, for any such 3-space we have $\partial \mathcal{K}(\mathcal{C}) \cap \Gamma \subset \partial \mathcal{K}(\mathcal{C} \cap T) \cap \Gamma$.

\footnotetext{
${ }^{12}$ This bound is tight if intersections are counted with multiplicities and over $\mathbb{P}^{2}(\mathbb{C})$.
} 
Proof. Since $\mathcal{K}(\mathcal{C})$ is convex, its intersection with $\Gamma$ is a (possibly empty) small great circle arc $\eta$. If $\eta$ is reduced to a single point then Lemma 3.2 implies that $\mathcal{C}$ has a unique transversal with direction in $\Gamma$, and any 3 -space $T$ containing this transversal will do. Otherwise, let $T$ be some ${ }^{13} 3$-space containing the two transversals to $\mathcal{C}$ with directions in $\partial \eta$. As $\mathcal{K}(\mathcal{C} \cap T)$ is convex, its intersection with $\Gamma$ is a small great circle arc. Since this arc contains $\partial \eta$ it contains $\eta$, and the other inclusion is immediate.

Let $T$ be a 3 -transversal to $\mathcal{C}$ such that $\mathcal{K}(\mathcal{C}) \cap \Gamma=\mathcal{K}(\mathcal{C} \cap T) \cap \Gamma$. By Lemma 3.2, the projections of $\mathcal{C}$ along any direction $\vec{u} \in \partial \mathcal{K}(\mathcal{C}) \cap \Gamma$ intersect in a single point; the projections of $\mathcal{C} \cap T$ along $\vec{u}$ must then also intersect in a single point, and $\vec{u} \in \partial \mathcal{K}(\mathcal{C} \cap T)$.

In particular, if the cone of directions of some sequence of disjoint balls $\mathcal{C}$ in $\mathbb{R}^{d}$ contains a great circle arc $\Gamma$ on its boundary, then $\Gamma$ also appears on the boundary of the section of $\mathcal{C}$ by some 3 -transversal; the strict convexity thus extends from the 3 -dimensional case to higher dimensions.

3.6. Immediate consequences. Let $\mathcal{C}$ be a finite collection of disjoint balls in $\mathbb{R}^{d}$. The following are simple consequences of Theorem 3.1 .

3.6.1. Topology of order-respecting transversals. Obviously, two transversals to $\mathcal{C}$ that realize distinct geometric permutations belong to different connected components of $\mathcal{T}_{1}(\mathcal{C})$. Theorem 3.1 implies that the converse is true [19, Lemma 14]:

THEOREM 3.2. The set of transversals to a finite number of disjoint balls in $\mathbb{R}^{d}$ in a given order is contractible.

Proof. Let $\mathcal{C}$ be a finite sequence of disjoint balls and $L$ its set of orderrespecting transversals. A transversal $\ell$ to $\mathcal{C}$ is barycentric if it goes through the center of mass of the intersection of the projections of the balls in $\mathcal{C}$ along $\vec{\ell}$. For any direction $v$ in $\mathcal{K}(\mathcal{C})$ there is a unique barycentric transversal to $\mathcal{C}$, which we denote $b_{\mathcal{C}}(v)$. Let $L^{*}$ denote the set of order-respecting barycentric transversals to $\mathcal{C}$. The projection of a ball changes continuously with the direction of projection, so $b_{\mathcal{C}}$ is continuous. Since the direction of a line changes continuously with the line, $b_{\mathcal{C}}^{-1}$ is also continuous and $b_{\mathcal{C}}$ defines a homeomorphism between $L^{*}$ and $\mathcal{K}(\mathcal{C})$. By Theorem 3.1, $\mathcal{K}(\mathcal{C})$ is convex and hence contractible. It follows that $L^{*}$ is also contractible. The map

$$
\left\{\begin{array}{l}
L \times[0,1] \rightarrow L \\
(\ell, t) \mapsto \ell+t\left(b_{\mathcal{C}}\left(v_{\ell}\right)-\ell\right)
\end{array}\right.
$$

is continuous and shows that $L^{*}$ is a deformation retract of $L$. Since $L^{*}$ is contractible, so is $L$. $\square$

3.6.2. Isotopy and geometric permutations. Two transversals to $\mathcal{C}$ are said to be isotopic if one can be moved continuously into the other while remaining a

\footnotetext{
${ }^{13}$ If the two transversals are skew, $T$ is unique.
} 
transversal during the motion, i.e. if they belong to the same path-connected component of $\mathcal{T}_{1}(\mathcal{C})$. Theorem 3.2 implies that the number of geometric permutations of $\mathcal{C}$ is equal to the number of connected components of $\mathcal{T}_{1}(\mathcal{C})$ :

COROLLARY 3.1. Two transversals to a finite family of disjoint balls in $\mathbb{R}^{d}$ are isotopic if and only if they induce the same geometric permutation.

Koltun and Sharir [55, Theorem 5.4] showed that the number of isotopy classes of transversals to $n$ disjoint balls is $O\left(n^{3+\epsilon}\right)$ for $d=3$ and $O\left(n^{2 d-2}\right)$ for $d \geq 4$; their proofs recast the set of transversals as a sandwich region in an arrangement of hyperplanes and builds on a series of results on the structure of such arrangements. With Corollary 3.1, Theorem 4.1 immediately improves these bounds.

3.6.3. Pinning configurations. Since $\mathcal{T}_{1}(\mathcal{C})$ can be recast as an union of cells in an arrangement of algebraic surfaces of bounded degree [55], it has a bounded number of connected components. Thus, a point in $\mathcal{T}_{1}(\mathcal{C})$ is isolated if and only if it is a connected component of $\mathcal{T}_{1}(\mathcal{C})$. Minimal pinning configurations can then be characterized as follows:

COROLLARY 3.2. Let $\ell$ be an order-respecting transversal to a finite sequence $\mathcal{C}$ of disjoint balls in $\mathbb{R}^{d} . \mathcal{C}$ pins $\ell$ if and only if no other transversal to $\mathcal{C}$ realizes the same geometric permutation as $\ell$, or equivalently:

$$
\mathcal{K}(\mathcal{C})=\{\vec{\ell}\} \quad \Leftrightarrow \quad \mathcal{K}^{o}(\mathcal{C})=\emptyset \quad \Leftrightarrow \quad \mathcal{K}\left(\mathcal{C}^{o}\right)=\emptyset .
$$

Proof. Since $\mathcal{C}$ pins $\ell$ if and only if $\{\ell\}$ is a connected component of $\mathcal{T}_{1}(\mathcal{C})$, the first equivalence follows from Theorem 3.2. If $\mathcal{K}(\mathcal{C})=\{\vec{\ell}\}$ then Lemma 3.2 ensures that no other line realizes the same geometric permutation as $\ell$, and the second equivalence follows. The remaining equivalences are straightforward. $\square$

Since a transversal is isolated if and only if no other transversal realizes the same geometric permutation, and two lines are always contained in some common 3 -space, we have:

COROLlaRY 3.3. A finite collection $\mathcal{C}$ of disjoint balls in $\mathbb{R}^{d}$ pins a line $\ell$ if and only if for every 3 -space $T$ that contains $\ell, \mathcal{C} \cap T$ pins $\ell$ in $T$.

4. Geometric permutations. The first investigation of geometric permutations is, to the best of our knowledge, due to Katchalski, Lewis and Liu [50]. Since then, the maximum number of geometric permutations was studied for a variety of different shapes: convex sets $[6,10,28,52,70,78]$, boxes [80], fat convex sets [54], translates of a convex set [8, 9, 11, 51], balls [73], congruent balls $[20,21,48,53,73]$, balls with bounded radius disparity [48, 81]. For disjoint balls, the bounds can be summarized as follows:

THEOREM 4.1. The maximum number of geometric permutations of a family of $n$ disjoint balls in $\mathbb{R}^{d}$ is $\Theta\left(n^{d-1}\right)$ if the balls have arbitrary radii, $O\left(\gamma^{\log \gamma}\right)$ if the balls have radius disparity at most $\gamma$, at most 3 if the balls have equal radii and at most 2 if, in addition, $n \geq 9$ or $n \geq 4$ and $d=2$.

The following description of the geometric permutations in the case of unit radius will be used in Section 5: 
THEOREM 4.2. Two geometric permutations of $n$ disjoint unit balls in $\mathbb{R}^{d}$, with $n \geq 9$ or $n \geq 4$ and $d=2$, differ by switching two adjacent elements.

These bounds were obtained through essentially three techniques we now review: separation sets, switch pairs and incompatible pairs.

4.1. Separation sets. Let $\mathcal{C}$ be a collection of disjoint convex sets in $\mathbb{R}^{d}$. A separation set $\mathcal{H}$ for $\mathcal{C}$ is a set of hyperplanes such that any two members in $\mathcal{C}$ can be separated by a hyperplane parallel to some element in $\mathcal{H}$. An oriented transversal $\ell$ to two disjoint convex sets $C_{1}$ and $C_{2}$ meets $C_{1}$ first if and only if for some hyperplane $\Pi$ separating $C_{1}$ and $C_{2}, \ell$ meets the halfspace containing $C_{1}$ first; in other words, if $\Gamma_{\Pi}$ denotes the hypersphere of directions of $\Pi$, it depends on which side of $\Gamma_{\Pi} \vec{\ell}$ lies. Thus, the geometric permutation realized by a transversal to $\mathcal{C}$ depends only on the cell of the arrangement on $\mathbb{S}^{d-1}$ of the hyperspheres of directions associated with the members of $\mathcal{H}$ that contains its direction. As a consequence, the number of geometric permutations of $\mathcal{C}$ is bounded by the complexity of that arrangement, that is $O\left(|\mathcal{H}|^{d-1}\right)$, and $n$ disjoint compact convex objects have $O\left(n^{2 d-2}\right)$ geometric permutations [78].

Upper bound for balls. Collections of disjoint balls admit small separation sets [73, Theorem 4.1]. The argument goes as follows. Let $\mathcal{C}=\left\{B_{1}, \ldots, B_{n}\right\}$ be a collection of disjoint balls in $\mathbb{R}^{d}$. Cover the sphere of directions $\mathbb{S}^{d-1}$ by spherical caps $C_{1}, \ldots, C_{k}$ of given opening angle $\alpha$. For any $1 \leq i \leq n$ and $1 \leq j \leq k$ let $\Gamma_{i, j}$ denote the cone with apex the center of $B_{i}$ induced by cap $C_{j}$ and $h_{i, j}$ a hyperplane separating $B_{i}$ from the closest ball with larger radius and having its center in $\Gamma_{i, j}$, if any; specifically, $h_{i, j}$ is chosen tangent to $B_{i}$ and normal to the line through the centers of the two separated balls (see Figure 5).

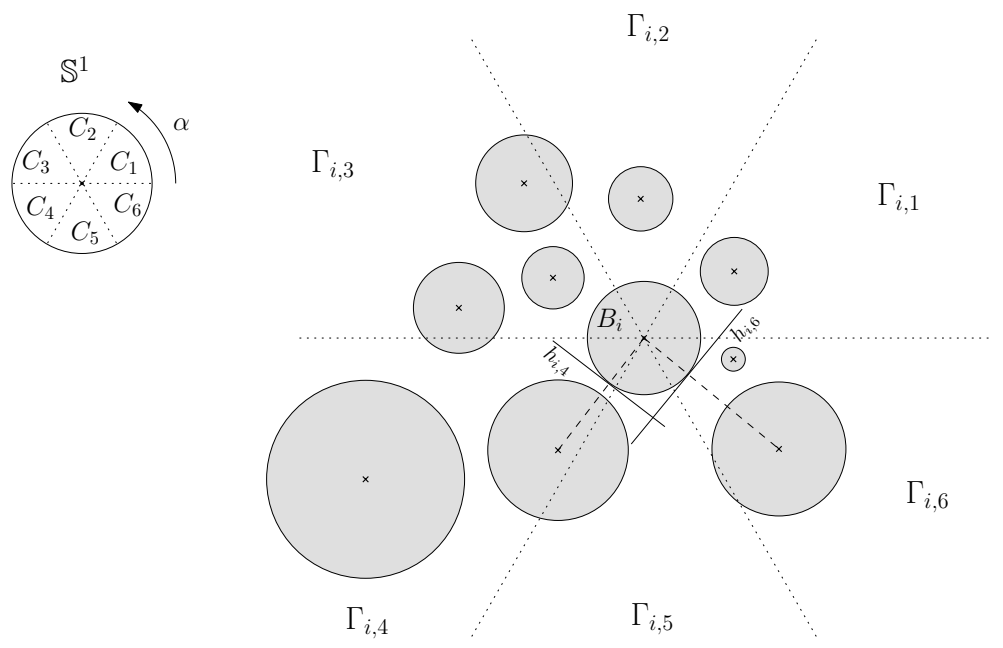

FIG. 5. Construction of a small separation set of a collection of balls $\left(d=2, k=6, \alpha=\frac{\pi}{3}\right)$. 
For $\alpha$ smaller than $\sin ^{-1}\left(\frac{\sqrt{3}-1}{2}\right)$, the collection

$$
\left\{h_{i, j} \mid 1 \leq i \leq n, 1 \leq j \leq k\right\}
$$

separates any two balls in $\mathcal{C}$. As a consequence, any collection of $n$ disjoint balls admits a separation set of size $O(n)$, and has $O\left(n^{d-1}\right)$ geometric permutations.

Lower bound for balls. The previous upper bound is asymptotically tight [73, Theorem 4.5]. Consider $n$ hyperplanes $H_{1}, \ldots, H_{n}$ in $\mathbb{R}^{d}$ going through the origin, no $d$ of them containing a line, and let $S_{i}$ denote the set of directions parallel to $H_{i}$. Let $\epsilon>0$ be small enough such that any cell in the arrangement $\mathcal{A}$ of $\left\{S_{1}, \ldots, S_{n}\right\}$ on $\mathbb{S}^{d-1}$ contains a point at distance at least $\epsilon$ from every $S_{i}$. For $\delta>0$, let $\left(B_{i}^{1}(\delta), B_{i}^{2}(\delta)\right)$ be two balls centered on the perpendicular to $H_{i}$ through the origin, at distance $\delta$ from the origin and separated by $H_{i} ; B_{i}^{1}(\delta)$ and $B_{i}^{2}(\delta)$ have equal radius, chosen such that a line through the origin intersects them if and only if it makes an angle at least $\epsilon$ with $H_{i}$. The construction consists of a pair $\left(B_{i}^{1}\left(\delta_{i}\right), B_{i}^{2}\left(\delta_{i}\right)\right)$ for $i=1, \ldots, n$, where $\delta_{1}=1$ and $\delta_{i+1}$ is chosen larger than the diameter of $\bigcup_{1 \leq t \leq i}\left(B_{t}^{1}\left(\delta_{t}\right) \cup B_{t}^{2}\left(\delta_{t}\right)\right)$. By construction, any line through the origin with direction at least $\epsilon$ away from each of the $S_{i}$ intersects all the balls. There are as many classes of such lines as cells in $\mathcal{A}$, that is $\Omega\left(n^{d-1}\right)$, and two lines with directions in different cells realize different orderings of the balls.

4.2. Switch pairs. Let $\mathcal{C}$ be a family of $n$ disjoint balls in $\mathbb{R}^{d}$ with radius disparity at most $\gamma$ that admits some transversal. Assume, w.l.o.g. that the radius of the smallest ball in $\mathcal{C}$ is 1 . For all asymptotic estimates we assume that $d$ is constant and $n \gg \gamma^{d-1}$.

For $n$ large enough, the transversals to $\mathcal{C}$ are nearly parallel. Specifically, a volume argument shows that the diameter of the set of centers of balls in $\mathcal{C}$ is $\Omega\left(\frac{n}{\gamma^{d-1}}\right)$; the angle between two transversals to $\mathcal{C}$ is then $O\left(\frac{\gamma^{d}}{n}\right)$ as the distance between them is at most $2 \gamma$ along segments of length $\Omega\left(\frac{n}{\gamma^{d-1}}\right)$. We say that two transversals are oriented consistently if the angle between their direction vectors is less than $\pi / 2$, that is, close to 0 when the transversals are nearly parallel. A switch pair for $\mathcal{C}$ is a pair of balls intersected in different orders by two transversals to $\mathcal{C}$ oriented consistently ${ }^{14}$. Switch pairs were investigated first for congruent disks in the plane $[72,73]$, then for balls in higher dimensions, both for the unit radius case $[20,48,53]$ and the bounded radius disparity case $[48,81]$.

4.2.1. Properties of a switch pair. For $n$ sufficiently large, a ball participates in at most one switch pair and two balls in a switch pair appear consecutively in any geometric permutation of $\mathcal{C}$ ([53, Lemmas 2.8 and 2.9], [48, Lemmas 8 and 9] and [81, Lemmas 2.10 and 2.11]). These properties follow from simple geometric considerations:

\footnotetext{
${ }^{14} \mathrm{~A}$ similar, but more general, notion is investigated by Asinowski et al. [9].
} 
- the distance between two balls in a switch pair is $O\left(\frac{\gamma^{2 d+1}}{n^{2}}\right)$ ([53, Lemmas 2.6 and 2.7], [48, Lemma 6] and [81, Lemma 2.7]),

- the line through the centers of the balls in a switch pair makes angle $\frac{\pi}{2}-O\left(\frac{\gamma^{d}}{n}\right)$ with any transversal to $\mathcal{C}$ ([53, Lemmas 2.6 and 2.7], [48, Lemma 6] and [81, Lemma 2.8]).

Consequently, to bound the number of geometric permutations of $\mathcal{C}$ it suffices to bound its number of switch pairs, as $k$ switch pairs allow at most $2^{k}$ geometric permutations.

4.2.2. Number of switch pairs. The number of switch pairs can be bounded via considerations on the distances between their inner centers of similitude. Let $\Delta$ be the line through the centers of the two balls furthest apart. The disjointedness of the balls and the upper bound on the distance between two members of a switch pair imply that the projection on $\Delta$ of the inner centers of similitude of two switch pairs are distance at least $\sqrt{2}-o(1)$ and at most $2 \gamma+o(1)$ apart ([81, Theorem 4.3], [53, Lemma 3.2] and [48, Lemma 16]). An upper bound of $1+\lfloor\sqrt{2} \gamma\rfloor$ on the number of switch pairs follows when $n$ is large enough. In the planar case, a different argument based on incompatible pairs (see Section 4.3) yields the same result [81].

4.2.3. Hamming distance between geometric permutations. The previous bound on the number of switch pairs yields that sufficiently large collections of disjoint balls with radius disparity $\gamma$ have at most $2^{1+\lfloor\sqrt{2} \gamma\rfloor}$ geometric permutations. Number the $m$ switch pairs of $\mathcal{C}$ and assign to every geometric permutation of $\mathcal{C}$ a vector in $\{0,1\}^{m}$ depending on the ordering in which each pair is traversed. If two geometric permutations differ by the switching of $k$ pairs then the radius disparity of the balls is at least $2^{\left\lceil\frac{k}{2}\right\rceil-1}$ [81]. Thus, the number of elements that differ, i.e. the Hamming distance, between the vectors of two geometric permutations is bounded by $2(1+\lfloor\log \gamma\rfloor)$. The size of a subset of $\{0,1\}^{m}$ with diameter at most $2 \delta$ under the Hamming distance is $O\left(\frac{(4 m)^{\delta}}{\delta !}\right)$. Therefore, disjoint balls with radius disparity at most $\gamma$ have $O\left(\gamma^{\log \gamma}\right)$ geometric permutations.

4.2.4. The case of unit balls. The previous result implies that sufficiently large collections of disjoint unit balls have at most 4 geometric permutations. This bound was reduced to 2 by ad hoc techniques.

In the plane. If $\mathcal{C}$ admits 3 geometric permutations it has at least two switch pairs, say $(A, B)$ and $(C, D)$. Up to symmetries we can then assume that the four disks admit the three geometric permutations $A B C D, B A C D$, and $A B D C$. Thus, the cells of the Voronoi diagram of the centers of these disks, where each cell inherits the label of the disk it contains, also admit these three geometric permutations. An elementary case-study of the configurations of four points in the plane shows that this is impossible [73]. Thus, any sufficiently large family of disjoint unit disks in $\mathbb{R}^{2}$ has at most one switch pair and at most 2 geometric permutations. 
In higher dimensions. A refined analysis shows that the distance between the inner centers of similitude of two switch pairs consisting of unit balls is at most $1+o(1)$ [20, Lemma 4]. Since this contradicts the lower-bound of $\sqrt{2}-$ $o(1)$ previously obtained for the same distance, it proves that sufficiently large collections of disjoint unit balls have at most one switch pair and 2 geometric permutations.

4.3. Incompatible pairs. An efficient way to bound the size of a set of permutations is to show that certain patterns cannot occur. Given two geometric permutations $g p_{1}$ and $g p_{2}$ of $\mathcal{C}$ and two permutations on $k$ elements $p_{1}$ and $p_{2}$, we say that $\left(p_{1}, p_{2}\right)$ is a sub-pattern of $\left(g p_{1}, g p_{2}\right)$ if the restriction of $\left(g p_{1}, g p_{2}\right)$ to some $k$ objects in $\mathcal{C}$ yields two permutations that are equal, up to relabelling and reversing, to $\left(p_{1}, p_{2}\right)$. Showing that certain pairs of permutations on four elements cannot occur as sub-patterns of pairs of geometric permutations of disjoint unit balls led to bounds that are tight in the plane [7] and almost tight in higher dimensions [21]. In particular, these bounds also apply to small families, unlike those obtained by studying switch pairs. The use of incompatible pairs for studying geometric permutations can be traced back to Katchalski et al. [51, Section 5], although they use a different presentation.

4.3.1. Families with incompatible pairs. To bound the number of geometric permutations of disjoint unit balls, the incompatible pairs investigated are of size 4 (for situations where larger families were considered see eg. $[8,10]$ ). The pairs of geometric permutations considered for disjoint unit balls are

$$
\begin{array}{ll}
a=(1234,2143), & b=(1234,1432), \\
c=(1234,1423), & d=(1234,3142),
\end{array}
$$

and Table 6 summarizes all 12 geometric permutations on $1, \ldots, 4$, divided into three rows. Any pair of permutations in row $\alpha$ is equal, up to relabelling and

\begin{tabular}{|c|cccc|}
\hline$\alpha$ & 1234 & 2143 & 1432 & 4123 \\
\hline$\beta$ & 1243 & 4312 & 1342 & 3124 \\
\hline$\gamma$ & 1324 & 3142 & 1423 & 4132 \\
\hline
\end{tabular}

FIG. 6. The 12 geometric permutations on $1, \ldots, 4$.

reversing, to one of $a$ or $b$ and row $\beta$ or $\gamma$ can be obtained from $\alpha$ by adequate relabelling. Therefore, any quadruple of objects for which $a$ and $b$ are incompatible has at most three geometric permutations, one from each row (the same holds if $b$ is replaced by $(1234,1423)$ [51]). Given three different geometric permutations $\sigma_{1}, \ldots, \sigma_{3}$ of $n \geq 4$ objects for which $a$ and $b$ are incompatible, there are always 3 objects to which the restrictions of the $\sigma_{i}$ differ [21, Lemma 1]; from there, one can prove that any family of $n$ objects for which $a$ and $b$ are incompatible has at 
most three geometric permutations [21, Lemma 2]. Note that pairs other than $a$ and $b$ can be used equivalently, for instance $a$ and $(1234,4123)$ [7].

Similar arguments yield that any family of $n$ objects for which the pairs $a, \ldots, d$ are incompatible has at most two geometric permutations that differ only by the swapping of two adjacent elements [21, Lemma 3]. Proving Theorems 4.1 and 4.2 thus reduces to showing that the pairs $a$ to $d$ are incompatible.

4.3.2. The planar case. Consider two intersecting transversals to four disks, and mark on each line one point from each disk. Different situations arise depending on which half-line each point belongs (there are 29 such configurations). A careful analysis of these situations shows that for families of disjoint unit disks the pairs $a$ and $b\left[51\right.$, Lemmas 1-3] and $c$ and $d[7]^{15}$ are incompatible ${ }^{16}$. A different proof, avoiding the discussion of the 29 configurations, was given later by the same authors [9].

4.3.3. Higher dimensions. A proof that pair $a$ is incompatible for disjoint unit balls in $\mathbb{R}^{d}$ can be obtained through elementary, although tedious, geometric observations [21, Section 4]; this analysis essentially refines the earlier proof that sufficiently large collections of disjoint unit balls have at most one switch pair [20], another way to formulate the incompatibility of $a$. The proof of incompatibility of $b, c$ and $d$ rests on the following crucial observation:

LEMMA 4.1 ([21], Lemma 7). Let $\vec{v}$ be a direction of a transversal to 3 disjoint unit balls in $\mathbb{R}^{d}$ and $\vec{u}$ the vector from the center of the first to the center of the last ball met by that transversal. Then $\angle(\vec{v}, \vec{u})<\pi / 4$.

To see that pair $b$ is incompatible, let $\overrightarrow{v_{1}}$ and $\overrightarrow{v_{2}}$ be two directions of transversals intersecting four disjoint unit balls in, respectively, the orders 1234 and 1432. Let $c_{i}$ denote the center of ball $i$. By Lemma 4.1 we have that $\angle\left(\overrightarrow{v_{1}},-\overrightarrow{v_{2}}\right)<\pi / 2$ since both $\overrightarrow{v_{1}}$ and $-\overrightarrow{v_{2}}$ make an angle less than $\pi / 4$ with $\overrightarrow{c_{2} c_{4}}$. Also, $\angle\left(\overrightarrow{v_{1}}, \overrightarrow{v_{2}}\right)<$ $\pi / 2$ as both $\overrightarrow{v_{1}}$ and $\overrightarrow{v_{2}}$ make an angle less than $\pi / 4$ with $\overrightarrow{c_{1} c_{3}}$, and we get a contradiction.

A packing argument ([21, Lemma 6]) shows that the intersection of the solids bounded by two cylinders of radius 1 whose axis make angle at least $\pi / 4$ contains at most 8 points with smallest inter-point distance at least 2 . Thus, two transversals to any collection of $n \geq 9$ disjoint unit balls in $\mathbb{R}^{d}$ make an angle of less than $\pi / 4$. Now, let $\vec{v}$ and $\overrightarrow{v^{\prime}}$ be the direction vectors of transversals to $n \geq 9$ disjoint unit balls that realize, respectively, the permutations 1234 and one of 1423 or 3142 on some subset of four balls. Because there are $n \geq 9$ balls,

$$
\angle\left(\vec{v}, \overrightarrow{v^{\prime}}\right)<\frac{\pi}{4}
$$

and Lemma 4.1 implies that the angle between $\vec{v}$ and $\overrightarrow{c_{2} c_{4}}$ is at most $\frac{\pi}{4}$. Consequently, the angle between $\overrightarrow{v^{\prime}}$ and $\overrightarrow{c_{2} c_{4}}$ is less than $\frac{\pi}{2}$ and the second line should

\footnotetext{
${ }^{15}$ The same result was obtained independantly by A. Holmsen in his master's thesis.

${ }^{16}$ For families of disjoint translates of a convex set, pairs $a$ and $b$ remain incompatible [51] but pairs $c$ and $d$ cannot be both incompatible: indeed, there exist arbitrarily large such families with three geometric permutations.
} 
meet ball 2 before ball 4 , a contradiction. Thus pairs $c$ and $d$ are incompatible for any family of $n \geq 9$ disjoint unit balls in $\mathbb{R}^{d}$. This proves Theorem 4.1 and Theorem 4.2 for $d \geq 3$.

5. Pinning, Hadwiger and Helly numbers. The Helly-type theorems for transversals to disjoint balls essentially generalize two landmark results in geometric transversal theory due to Hadwiger and Danzer.

Hadwiger's transversal theorem states that $n$ disjoint ${ }^{17}$ convex sets in the plane have a transversal if any 3 have a transversal consistent with some global ordering of the family [41]. The bound on the Hadwiger number shows that this theorem generalizes to disjoint balls in arbitrary dimension, a situation that is remarkable as it is not the case for disjoint translates of a convex set, not even in $\mathbb{R}^{3}[46]$.

Danzer proved that $n$ disjoint unit disks ${ }^{18}$ in the plane have a transversal if any 5 do [23], and conjectured that a similar result holds in higher dimensions. The bound on the Helly number for disjoint unit balls is the positive answer to this question.

5.1. Relationship between the pinning and Hadwiger numbers. In the plane, the pinning and Hadwiger numbers are the same, namely 3. In higher dimensions, the convexity of the cone of directions (Theorem 3.1) implies:

THEOREM 5.1. If $p_{d}$ and $h_{d}$ denote respectively the pinning and Hadwiger numbers of collections and sequences of disjoint balls in $\mathbb{R}^{d}$ then $h_{d} \leq p_{d}+1$.

Proof. Let $\mathcal{C}$ be a sequence of at least $n \geq p_{d}+2$ disjoint balls and assume that every subsequence of size $p_{d}+1$ has an order-respecting transversal. Shrink continuously all balls by, for instance, multiplying all radii by some parameter $t$ ranging from 1 down to 0 , until some subsequence $X$ of size $p_{d}+1$ is about to lose its last order-respecting transversal. By Theorem 3.1, at that position $\mathcal{K}(X)$ is a single point and $X$ has a unique order-respecting line transversal $\ell$. Since $(X, \ell)$ is a pinning configuration, there exists a subset $Y \subset X$ of size at most $p_{d}$ such that $Y$ pins $\ell$. Given any $Z \in(\mathcal{C} \backslash Y)$, the subsequence $Y \cup\{Z\}$ has size at most $p_{d}+1$ and thus has some order-respecting line transversal $\ell_{Z}$. Since $(Y, \ell)$ is a pinning configuration, $Y$ admits no order-respecting transversal other than $\ell$, and thus $\ell_{Z}=\ell$ and $\ell$ intersects $Z$. It follows that $\mathcal{C}$ has a line transversal, and $h_{d} \leq p_{d}+1$. $\square$

REMARK 5.1. The same proof yields that if every subsequence of size $p_{d}+2$ has an order-respecting transversal then $\mathcal{C}$ has an order-respecting transversal.

5.2. Bounds on the pinning and Hadwiger numbers. The current bounds on the pinning and Hadwiger numbers, given by Theorem 5.3, grow linearly with the dimension [19]. We sketch the proof of these bounds, after giving a much simpler argument that yields a bound quadratic in the dimension [3, 40, 45].

\footnotetext{
${ }^{17}$ This assumption can be dropped [77].

${ }^{18}$ Grünbaum proved the same statement for unit axis-parallel squares [37], and conjectured that it holds for collections of disjoint translates of a convex set, a conjecture proven 30 years later by Tverberg [75].
} 
5.2.1. A simple quadratic bound. The pinning and Hadwiger numbers can be bounded by applying Helly's theorem successively on $\mathbb{S}^{d}$ and on the projections along certain directions, an argument already used by Vincensini [76].

LEMMA 5.1. The pinning and Hadwiger numbers of disjoint balls in $\mathbb{R}^{d}$ are bounded from above by $d(d+1)$.

Proof. Let $\mathcal{C}$ be a sequence of disjoint balls in $\mathbb{R}^{d}$ and $\left(\begin{array}{l}\mathcal{C} \\ d\end{array}\right)$ the set of its subsequences of length $d$. As argued in the proof of Lemma 3.1,

$$
\mathcal{K}(\mathcal{C})=\bigcap_{X \in\left(\begin{array}{c}
\mathcal{C} \\
d
\end{array}\right)} \mathcal{K}(X)
$$

and Theorem 3.1 yields that for any subsequence $X$ the set $\mathcal{K}(X)$ is strictly convex.

The spherical Helly theorem on $\mathbb{S}^{d-1}$ (Theorem 2.2) implies that $\mathcal{K}(\mathcal{C})$ is nonempty if and only if for any $d+1$ elements $X_{1}, \ldots, X_{d+1} \in\left(\begin{array}{l}\mathcal{C} \\ d\end{array}\right)$ the intersection $\bigcap_{1 \leq i \leq d+1} \mathcal{K}\left(X_{i}\right)$ is nonempty. In other words, $\mathcal{C}$ has a transversal ${ }^{19}$ if and only if any subsequence of length at most $d(d+1)$ has an order-respecting line transversal. This proves the statement for the Hadwiger number.

Similarly, $\mathcal{K}^{o}(\mathcal{C})$ is the intersection of the $\mathcal{K}^{o}(X)$ for $X \in\left(\begin{array}{l}\mathcal{C} \\ d\end{array}\right)$. Thus, if $\mathcal{C}$ pins some order-respecting transversal $\ell$, the same arguments yield that $\mathcal{K}^{\circ}(\mathcal{C})$ is empty if and only if $\mathcal{K}^{\circ}(X)$ is empty for some subsequence $X \subset \mathcal{C}$ of length at most $d(d+1)$. Since $\mathcal{K}(\mathcal{C}) \subset \mathcal{K}(X)$ we deduce that $\mathcal{K}(X)$ is a single point, and $X$ pins $\ell$ as well. This proves the statement for the pinning number.

REMARK 5.2. If the balls are unit this bound becomes $d^{2}$ : by Lemma 4.1, the cone of directions of any sequence of $n \geq 3$ balls has opening angle at most $\frac{\pi}{4}$, so we can apply Helly's theorem in $\mathbb{R}^{d-1}$ instead of $\mathbb{S}^{d-1}$ (Theorem 2.3 instead of Theorem 2.2) in the previous proof.

5.2.2. A linear bound. For thinly distributed balls, Grünbaum [38] obtained a linear bound on the Hadwiger number by applying Helly's topological theorem directly to the set of line transversals. More generally ${ }^{20}$, we have:

THEOREM 5.2. Let $\mathcal{U}_{d}$ be the set of all collections of balls in $\mathbb{R}^{d}$ admitting a separation set of size 1 . The pinning, Hadwiger and Helly numbers of $\mathcal{U}_{d}$ are bounded from above by $2 d-1$.

Proof. Let $\mathcal{C}=\left\{B_{1}, \ldots, B_{n}\right\}$ be a sequence of balls in $\mathbb{R}^{d}$ with separation set $\{H\}$ (in particular the balls are pairwise disjoint). Let $\epsilon$ denote the minimal angle any transversal to two balls in $\mathcal{C}$ makes with $H$, and let $T(X)$ denote the set of transversals to a subsequence $X \subset \mathcal{C}$ making an angle at least $\epsilon$ with $H$. Parameterizing lines by their intercept in two translated copies of $H$ recasts the $T\left(B_{i}\right)$ as contractible subsets of $\mathbb{R}^{2 d-2}$. Thus, to apply Helly's topological theo-

\footnotetext{
${ }^{19}$ In fact, $\mathcal{C}$ has an order-respecting transversal.

${ }^{20}$ Grünbaum's proof exploits the fact that the distance condition that characterizes thinly distributed balls guarantees that the family has a separation set of size 1 .
} 
rem it suffices to prove:

$$
\forall A_{1}, \ldots, A_{2 d-2} \in \mathcal{C}, \quad \bigcap_{1 \leq i \leq 2 d-2} T\left(A_{i}\right) \text { is a homology cell. }
$$

Since $\mathcal{C}$ has a separation set of size one, any subsequence of $\mathcal{C}$ has at most one geometric permutation. Therefore, for $X, Y \subset \mathcal{C}$ we have that

$$
T(X) \cap T(Y)=T(X \cup Y)
$$

and the above condition follows from Theorem 3.2. Therefore, the Helly number for thinly distributed balls in $\mathbb{R}^{d}$ is at most $2 d-1$. Because all subsequences have a unique geometric permutation, the bound on the Hadwiger number follows. Since a transversal is isolated if the open balls have no transversal in the same order, the bound on the pinning number also follows. $\square$

Compatible directions. The same idea can be applied to more general families of balls by restricting the set of possible directions of transversals so that any subsequence of $\mathcal{C}$ has only one geometric permutation [19]. Specifically, call a direction $\vec{u}$ compatible with a sequence $\mathcal{C}$ if

$$
\forall A \prec B \text { in } \mathcal{C}, \quad\langle\vec{u}, \overrightarrow{a b}\rangle>0,
$$

where $a$ and $b$ denote the respective centers of $A$ and $B$; by extension, we say that a transversal to $X \subset \mathcal{C}$ is compatible if its direction is. The directions of compatible transversals to a subsequence $X \subset \mathcal{C}$ are the intersection of $\mathcal{K}(X)$ with a polytope in $\mathbb{S}^{d-1}$, and thus strongly convex; the same proof as in Theorem 3.2 yields that the set of compatible transversals to $X \subset \mathcal{C}$ is contractible. Also, a compatible transversal to $X, Y \subset \mathcal{C}$ is order-respecting on $X \cup Y$. As a consequence, the proof of Theorem 5.2 yields [19, Lemma 15]:

LEMMA 5.2. If $\mathcal{C}$ is a sequence of disjoint open balls such that any subset of size $2 d-1$ has a transversal compatible with $\mathcal{C}$, then $\mathcal{C}$ has a compatible transversal.

We can now bound the pinning and Hadwiger numbers [19, Proposition 13]:

THEOREM 5.3. The pinning and Hadwiger numbers for disjoint balls in $\mathbb{R}^{d}$ are bounded from above by, respectively, $2 d-1$ and $2 d$.

Proof. Let $\mathcal{C}$ be a sequence of disjoint balls in $\mathbb{R}^{d}$ that pins an order-respecting transversal $\ell$. From Corollary 3.2 we get that the open balls in $\mathcal{C}$ have no compatible transversal, and so Lemma 5.2 yields that some subsequence $X \subset \mathcal{C}$ of size at most $2 d-1$ has no transversal compatible with $\mathcal{C}$. Since $\mathcal{K}(X)$ is convex and the set of compatible directions, which is open, intersects $\mathcal{K}(X)$ but not $\mathcal{K}^{\circ}(X), \mathcal{K}(X)$ has empty interior and $X$ pins $\ell$. Thus, the pinning number is at most $2 d-1$ and Theorem 5.1 bounds the Hadwiger number by $2 d$.

5.3. Helly numbers. A family of examples by Danzer [23] (see also [44, Figure 3]) shows that the Helly number of disjoint balls is already unbounded in dimension 2. It can, still, be bounded under additional assumptions, e.g. for thinly 
distributed balls (Theorem 5.2). The case of congruent balls received particular attention and the bounds can be summarized as follows:

THEOREM 5.4. The Helly number of families of disjoint unit balls in $\mathbb{R}^{d}$ is 5 for $d=2$ and at most $4 d-1$ for $d \geq 3$.

We describe in Sections 5.3.1 and 5.3.2 the two techniques used to obtain such bounds. We show in Section 5.3.3 that the requirement that the balls be unit can be replaced by a bound on the number of geometric permutations of all subfamilies (Theorem 5.6). This implies, for instance, that the Helly number of a family of balls with radius disparity at most $\gamma$ can be bounded by a function of $d$ and $\gamma$ (Corollary 5.1).

5.3.1. Designing an ordering. Holmsen et al. [45] used the earlier analysis of switch pairs $[20,48,53,81]$ to bound the Helly number of disjoint unit balls in 3 dimensions by 22 .

Let $\delta$ denote the smallest diameter of a set of centers of 31 disjoint unit balls in $\mathbb{R}^{3}$ and $\mathcal{C}=\left\{B_{1}, \ldots, B_{n}\right\}$ a collection of at least 31 disjoint unit balls in $\mathbb{R}^{3}$. Assume that the centers of $B_{1}$ and $B_{n}$ are the furthest apart and let $\mathcal{T}$ denote the set of transversals to these two balls, oriented from $B_{1}$ to $B_{n}$. Say that $\left(B_{i}, B_{j}\right)$ is a switch pair if there are transversals in $\mathcal{T}$ that meet these two balls in distinct orders $^{21}$. A result similar to Theorem 4.2 applies to families with less than 9 balls provided the centers are sufficiently spread out [45, Theorem 3]:

LEMMA 5.3. Any family of disjoint unit balls in $\mathbb{R}^{3}$ whose set of centers has diameter at least $\delta$ has at most two switch pairs; the balls of a switch pair appear consecutively in any geometric permutation of the family.

We can then bound the Helly number as follows [45, Theorem 1]:

PROPOSITION 5.1. The Helly number of collections of at least 31 disjoint unit balls in $\mathbb{R}^{3}$ is at most 22 .

Proof. Assume that every subset of $\mathcal{C}$ of size at most 22 has a transversal. We discuss the case where $\mathcal{C}$ has two switch pairs $P_{1}$ and $P_{2}$ (if $\mathcal{C}$ has one or no switch pair the proof is similar). Lemma 5.3 implies that there exists an ordering $\prec^{\prime}$ on $\mathcal{C}^{\prime}=\mathcal{C} \backslash\left(P_{1} \cup P_{2}\right)$ such that any transversal in $\mathcal{T}$ to a subset of $\mathcal{C}^{\prime}$ respects $\prec^{\prime}$. Since the balls in each switch pair are consecutive, there are only 4 possible extensions of $\prec^{\prime}$ into an ordering of $\mathcal{C}$, say $\prec_{1}, \ldots, \prec_{4}$. Assume that for each $i=1, \ldots, 4$ there is a quadruple $Q_{i} \subset \mathcal{C}^{\prime}$ such that $Q_{i} \cup P_{1} \cup P_{2}$ has no transversal in $\mathcal{T}$ respecting $\prec_{i}$. Then the at most 22 balls of the subset

$$
\left(\bigcup_{1 \leq i \leq 4} Q_{i}\right) \bigcup P_{1} \bigcup P_{2} \bigcup\left\{B_{1}, B_{n}\right\}
$$

have no common transversal, which contradicts the assumption. Consequently, some extension $\prec_{i}$ of $\prec$ is such that for any quadruple $Q \subset \mathcal{C}^{\prime}$ the balls in $Q \cup$ $P_{1} \cup P_{2}$ have a transversal in $\mathcal{T}$ respecting $\prec_{i}$. It follows that every 6-tuple in $\mathcal{C}$ has a transversal respecting $\prec_{i}$, and since the Hadwiger number of disjoint balls in $\mathbb{R}^{3}$ is at most 6 (by Theorem 5.3), we get that $\mathcal{C}$ has a transversal.

\footnotetext{
${ }^{21}$ Note that this definition slightly differs from that used in Section 4.
} 
REMARK 5.3. This approach extends naturally to higher dimensions, resulting on a bound of $4 h_{d}-2$ for the Helly number of sufficiently large families of disjoint unit balls, where $h_{d}$ is the corresponding Hadwiger number. The threshold above which a family of balls is "sufficiently large" increases with the dimension.

REMARK 5.4. Holmsen et al. [45] used a bound of 12 on the Hadwiger number of disjoint balls in $\mathbb{R}^{3}$, thus obtaining a bound of 46 on the Helly number. Their theorem thus omits the assumption that the family be large enough.

5.3.2. The homotopy method. The technique used to bound the Hadwiger number in terms of the pinning number in Theorem 5.1 can also be used to bound the pinning number of disjoint unit balls [19, Theorem 2]:

THEOREM 5.5. The Helly number for disjoint unit balls in $\mathbb{R}^{d}, d \geq 2$, is bounded from above by $2 p_{d}+1$, where $p_{d}$ denotes the pinning number, for disjoint balls in $\mathbb{R}^{d}$.

Proof. Let $\mathcal{C}$ be a collection of disjoint unit balls in $\mathbb{R}^{d}$ such that any subset of $2 p_{d}+1$ balls has a transversal, where $p_{d}$ denotes the pinning number for disjoint (unit) balls in $\mathbb{R}^{d}$. Shrink uniformly the balls in $\mathcal{C}$ until the first subset of $2 p_{d}+1$ balls, say $\mathcal{F}$, is about to lose its last order-respecting transversal. Any subset of $\mathcal{F}$ of size at least $|\mathcal{F}|-2$ has at most two geometric permutations differing by the switching of two consecutive balls ${ }^{22}$. In the rest of this proof all balls are considered shrunk.

We first argue that we can assume that $\mathcal{F}$ has a unique transversal $\ell$. Otherwise, $\mathcal{F}$ has only isolated transversals, each one corresponding to a distinct geometric permutation. Theorem 4.2 yields that there are 2 such lines, say $\ell_{1}$ and $\ell_{2}$. Each $\ell_{i}$ can be pinned by $p_{d}$ balls from $\mathcal{F}$, so some subset $\mathcal{F}^{\prime} \subset \mathcal{F}$ of size $|\mathcal{F}|-1$ suffices to pin both of them and Theorem 4.2 implies that this subset has no other transversal. Consequently, every ball in $\mathcal{C} \backslash \mathcal{F}^{\prime}$ meets one of the $\ell_{i}$. If all such balls meet $\ell_{2}$, it is a transversal to $\mathcal{C}$ and we are done; if some ball $A$ misses $\ell_{2}$ then $\mathcal{F}^{\prime} \cup\{A\}$ is a subset of size at most $|\mathcal{F}|$ with $\ell=\ell_{1}$ as unique transversal.

Next, we argue that some proper subset $\mathcal{F}^{\prime}$ of $\mathcal{F}$ has no other transversal than $\ell$. Otherwise, let $\mathcal{G}$ be a subset of size $p_{d}$ that pins $\ell$ and for $Z \in \mathcal{F} \backslash \mathcal{G}$ denote by $\ell_{Z}$ a transversal to $\mathcal{F} \backslash\{Z\}$ other than $\ell$. Since $\mathcal{G}$ pins $\ell$, the orderings $\prec_{\ell}$ and $\prec_{\ell_{Z}}$ differ on $\mathcal{G}$ and thus on $\mathcal{F} \backslash\{Z\}$ and, by Theorem 4.2, they differ by the swapping of two balls $X_{Z}$ and $Y_{Z}$. Since $\prec_{\ell}$ and $\prec_{\ell_{Z}}$ already differ on $\mathcal{G}$, we have that $X_{Z}, Y_{Z} \in \mathcal{G}$. For $A, B \in \mathcal{F} \backslash \mathcal{G}$, the set $\mathcal{F} \backslash\{A, B\}$ has three transversals $(\ell$, $\ell_{A}$ and $\ell_{B}$ ) but, by Theorem 4.2, at most two geometric permutations. Since $\prec \ell$ and $\prec_{\ell_{Z}}$ disagree on $\mathcal{G}$, we thus get that $\ell_{A}$ and $\ell_{B}$ induce the same geometric permutation on $\mathcal{F} \backslash\{A, B\}$ for any $A, B \in \mathcal{F} \backslash \mathcal{G}$. It follows that $X_{Z}$ and $Y_{Z}$ are independent of the choice of $Z$; call these two balls $X$ and $Y$ and let $\prec$ be the ordering on $\mathcal{F}$ obtained by swapping $X$ and $Y$ in $\prec_{\ell}$. Since $\mathcal{F}$ has no transversal in the order $\prec$, Remark 5.1 implies that some subset $\mathcal{H} \subset \mathcal{F}$ of size $p_{d}+2$ has no transversal respecting that order. Thus, $\mathcal{F}^{\prime}=\mathcal{G} \cup \mathcal{H}$ has no other transversal than

\footnotetext{
${ }^{22}$ Theorem 4.2 applies as $p_{2}=3$ and $p_{d} \geq 5$ for $d \geq 3$ (see Section 5.4)
} 
$\ell$; the balls $X$ and $Y$ both belong to $\mathcal{H}$ as otherwise $\prec$ and $\prec_{\ell}$ are equivalent, so $\mathcal{F}^{\prime}$ has size at most $2 p_{d}$ and is a proper subset of $\mathcal{F}$.

Let $X$ be some ball in $\mathcal{C}$. Since $\mathcal{F}^{\prime} \cup\{X\}$ has some transversal and $\ell$ is the only transversal to $\mathcal{F}^{\prime}$, it follows that $\ell$ intersects $X$. Thus, $\mathcal{C}$ has a transversal.

Note that this bound is not tight in the two-dimensional case [23].

5.3.3. Using Helly's theorem for unions of sets. Using Matousek's generalization of Helly's topological theorem (see Theorem 2.6), we can replace the constraint on the radii radii by considerations on numbers of geometric permutations:

THEOREM 5.6. For any $d \geq 2$ and $k \geq 1$ there exists a number $h^{\star}(d, k)$ with the following property. Let $\mathcal{C}$ be a finite family of disjoint open balls in $\mathbb{R}^{d}$ such that any sub-family of $\mathcal{C}$ has at most $k$ geometric permutations. Then $\mathcal{C}$ has a line transversal if and only if every sub-family of size at most $h^{\star}(d, k)$ has a line transversal.

Proof. Let $\mathcal{C}=\left\{B_{1}, \ldots, B_{n}\right\}$ be a family of disjoint balls in $\mathbb{R}^{d}$ and $T_{i}$ the set of oriented line transversals to $B_{i}$. By Theorem 3.2, the intersection of any number of $T_{i}$ consists of at most $k$ contractible components. The $T_{i}$ are subsets of the Grassmaniann $G_{2,2 d-2}$, which naturally embeds in $\mathbb{P}^{2 d-1}$; to apply Theorem 2.6, we embed (part of) the $T_{i}$ in $\mathbb{R}^{2 d-2}$.

We handle this technicality as follows. Let $\Pi$ and $\Pi^{\prime}$ be two parallel planes and $T_{i}^{*}$ denote the set of oriented line transversals to ball $B_{i}$ that are not parallel to $\Pi$. By parameterizing all lines not parallel to $\Pi$ using their intersections with $\Pi$ and $\Pi^{\prime}$, we recast the $T_{i}^{*}$ as subsets of $\mathbb{R}^{2 d-2}$. The directions of lines in $T_{i}^{*}$ are exactly the directions of lines in $T_{i}$ minus a great hypersphere. Similarly, the directions of an intersection of $T_{i}^{*}$ consists in the difference of at most $k$ convex sets and a great hypersphere, which is at most $2 k$ convex sets. This implies that the intersection of any $T_{i}^{*}$ has at most $2 k$ connected components, and Theorem 2.6 applies. With $h^{\star}(d, k)=h(2 d-2,2 k)$, we thus get that if every subset of $\mathcal{C}$ of size $h^{\star}(d, k)$ has a line transversal not parallel to $\Pi$ then $\mathcal{C}$ has a line transversal. Now, observe that if a subset has a strict line transversal, then its cone of directions has nonempty interior and it must have a line transversal not parallel to $\Pi$.

By Theorem 4.1, this applies to case of balls of bounded radius disparity immediately:

COROLLARY 5.1. The Helly number of a family of disjoint balls in $\mathbb{R}^{d}$ with radius disparity at most $\gamma$ can be bounded by a function of $d$ and $\gamma$.

REMARK 5.5. Unfolding the same approach using Amenta's generalization of Helly's theorem (see Corollary 2.1) requires to control the intersection of a set $\mathcal{F}$ of directions of transversals to d-tuples of balls. If $\mathcal{F}$ is the set of all $d$-tuples of a family of balls, then this intersection consists of at most $k$ disjoint convex sets on the sphere $\mathbb{S}^{d-1}$. If $\mathcal{F}$ consists of some but not all d-tuples of a family of balls, the components of this intersection are still convex, but it is not clear what their number is.

5.4. Lower bounds. This section discusses the few lower bounds known for the pinning, Hadwiger and Helly numbers. First, we observe that these numbers 
are monotone in the dimension:

THEOREM 5.7. The pinning, Hadwiger and Helly numbers of disjoint balls in $\mathbb{R}^{d}$ are nondecreasing in $d$.

Proof. Let $\mathcal{C}$ be a collection of balls in $\mathbb{R}^{d}$ such that all centers lie in some $k$-flat $\Pi$. If $\ell$ is a transversal to $\mathcal{C}$ then so is the orthogonal projection of $\ell$ on $\Pi$, as the orthogonal projection reduces the distance to the balls' centers. As a consequence, any lower-bound example for the pinning, Hadwiger and Helly numbers in $\mathbb{R}^{k}$ can be embedded in $\mathbb{R}^{d}$ for $d \geq k$ while retaining its transversal properties, and these numbers are nondecreasing with $d$.

Two-dimensional examples. In the plane, the pinning and Hadwiger numbers of disjoint disks are at most 3 ; these bounds are easily seen to be tight (c.f. Figure 7(a) and (b)). Also, the Helly number of disjoint unit disks is exactly 5 , as

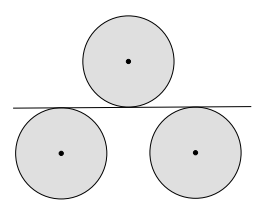

(a)

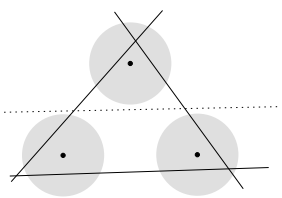

(b)

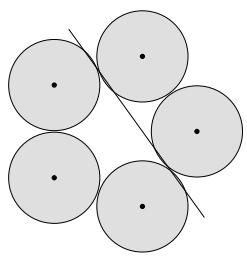

(c)

FIG. 7. Lower bounds in the plane for the pinning (a), Hadwiger (b) and Helly (c) numbers.

follows from the example of five unit disks centered at the vertices of a regular pentagon, depicted by Figure 7(c) (see [23, 44] for a more detailed description).

Higher dimensions. The upper bound for the pinning number of families of disjoint balls can be shown to be tight [18]:

THEOREM 5.8. The pinning number of disjoint balls in $\mathbb{R}^{d}$ is exactly $2 d-1$.

Call a pinning configuration $(\mathcal{C}, \ell)$ stable if $\ell$ remains pinned when the balls in $\mathcal{C}$ are perturbed by any sufficiently small (distinct) motions that keep $\ell$ fixed. Theorem 5.8 follows from two observations: (i) in any dimension there exists a finite stable pinning configuration (Figure 7 gives an example for $d=3$ ), and (ii) in $\mathbb{R}^{d}$, any stable pinning configuration has size at least $2 d-1$.

Theorem 5.8 also narrows the gap on the Hadwiger numbers:

COROLLARY 5.2. The Hadwiger number of disjoint balls in $\mathbb{R}^{d}$ is $2 d-1$ or $2 d$.

Since the example of Figure 7(a) can be embedded in $\mathbb{R}^{3}$, we also have that not every minimal pinning configuration has the same size.

6. Algorithmic aspects. The problem of computing a line transversal to some given collection of sets, if one exists, has been studied in a variety of situations: segments in the plane [15, 30, 62] and higher dimensions [13], convex polygons in the plane $[15,22]$, polyhedra in three dimensions [13, 49], translates of a convex set in the plane [29]. For families of balls, the best algorithms have 


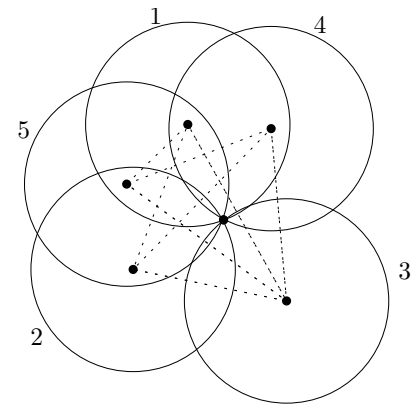

FIG. 8. Any configuration of 5 disjoint balls whose projection along a common tangent is as in the figure is a stable pinning configuration in $\mathbb{R}^{3}$.

complexity $O(n)$ for $n$ disjoint unit disks in the plane [4, 29], $O(n \log n)$ for $n$ intersecting unit disks in the plane [29] and $O\left(n^{3+\epsilon}\right)$ for $n$ balls in three dimensions [1]; if the dimension is part of the input, deciding if (intersecting) unit balls have a transversal is NP-hard [59]. We complete these results by:

THEOREM 6.1. A transversal to $n$ disjoint balls with bounded radius disparity in $\mathbb{R}^{d}$ can be computed in randomized $O(n)$ time.

This is remarkable as there is a $\Omega(n \log n)$ lower bound for this problem for $n$ segments or $n$ unit disks in the plane [12]. The constant in the $O()$ notation depends on the dimension and the radius disparity. The same holds, in any fixed dimension, for thinly distributed collections of balls or more generally any collection for which the number of geometric permutations of any sub-family is bounded.

The next sections briefly recalls the class of LP-type problems and uses the connection it bears to Helly-type theorems [4] for deducing Theorem 6.1 from Theorem 5.4.

6.1. Generalized linear programming. The linear programming problem, one of the fundamental problems in optimization, consists in maximizing some linear function while satisfying a family of linear equalities and inequalities. Geometrically, it translates into finding a point extremal in some direction (the gradient of the linear function) inside a polytope given as the intersection of halfspaces. Techniques for solving linear programming such as the randomized incremental algorithm of Seidel [69] have been known to solve other problems, for instance computing the smallest enclosing circle of a planar point set. This observation was formalized by Sharir and Welzl [71] in the framework of LP-type problems.

Let $\mathcal{H}$ be a set. Given $F \subset \mathcal{H}$ and $x \in \mathcal{H}$ we denote by $F+x$ and $F-x$ respectively the union and the difference of $F$ and $\{x\}$. An LP-type problem is a pair $(\mathcal{H}, w)$ consisting of a set $\mathcal{H}$ and a map $w: 2^{\mathcal{H}} \rightarrow \Omega$, where $\Omega$ is a totally ordered set with maximal element $N$, that satisfies for any $F \subset G \subset \mathcal{H}$ and $x \in \mathcal{H}$ the two properties: 
- Monotonicity: $w(F) \leq w(F+x)$.

- Locality: if $w(F)=w(G)$ then

$$
w(F+x) \neq w(F) \Leftrightarrow w(G+x) \neq w(G) .
$$

A subset $F \subset \mathcal{H}$ is a basis if it contains no proper subset with the same image under $w$ :

$$
\forall x \in F, \quad w(F-x)<w(F) .
$$

Any set $F \subset \mathcal{H}$ contains a basis $B$ with $w(B)=w(F) ; B$ is called a basis of $F$. A basis $B$ is feasible if $w(B)<N$. The combinatorial dimension of an LP-type problem is the maximal cardinality of a feasible basis. Sharir and Welzl [71] showed that if the combinatorial dimension of an LP-type problem $(\mathcal{H}, w)$ is bounded independently of $|\mathcal{H}|$, then a basis of $\mathcal{H}$ can be computed in randomized $O(|\mathcal{H}|)$ time [71].

If $(\mathcal{H}, w)$ is an LP-type problem of combinatorial dimension $k$, then $w(\mathcal{H})$ is equal to $w(B)$ for some subset $B \subset \mathcal{H}$ of size at most $k$; thus, for any $\lambda \in \mathbb{R}$, any LP-type problem satisfies the following Helly-type theorem:

$$
w(\mathcal{H}) \leq \lambda \text { if and only if }{ }^{23} w(B) \leq \lambda \text { for any } B \subset \mathcal{H} \text { of size at most } k .
$$

This connection goes, in fact, both ways [4] and a large class of Helly-type theorems have a corresponding LP-type problem. The next section applies this correspondence to Theorem 5.4.

6.2. LP-type formulation. Let $\mathcal{C}$ be a collection of disjoint closed balls in $\mathbb{R}^{d}$. Given a ball $X$ of radius $r$ and a real $\rho \geq 0$ we denote by $\rho X$ the ball with same center as $X$ and radius $\rho r$; given a collection $F$ of balls we also denote by $\rho F$ the collection $\{\rho X \mid X \in F\}$. Let $\Omega=[0,1] \cup\{N\}$ where $N$ is maximal and the order on $[0,1]$ is the natural one. The map

$$
\phi:\left\{\begin{aligned}
2^{\mathcal{C}} & \rightarrow \Omega \\
F & \mapsto \min (\{\rho \in[0,1] \mid \rho F \text { has a transversal }\} \bigcup\{N\})
\end{aligned}\right.
$$

associates to every sub-collection of balls the amount by which these balls can be "deflated" and still retain some transversal - possibly $N$ if the sub-collection had no transversal to begin with. Theorem 3.1 implies that if $\phi(F)<N$ then $\phi(F) F$ has only finitely many transversals. If $\mathcal{C}$ is not in generic position, there may be more than one such transversal and this implies that $(\mathcal{C}, \phi)$ may violate the locality condition (see Figure 9). Simply put, the system of transversals to balls doesn't meet the "unique minimum property" of Amenta [4]. This can be taken care of as follows ${ }^{24}$. Let $\nu(F)$ denote the number of transversals to $\phi(F) F$, with the convention that $\nu(F)=0$ whenever $\phi(F)=N$. Define $\Omega^{\prime}$ as $([0,1] \times \mathbb{Z}) \cup$ $\{(N, 0)\}$, ordered lexicographically, and $w=(\phi, \nu): 2^{\mathcal{C}} \rightarrow \Omega^{\prime}$.

LemMa 6.1. $(\mathcal{C}, w)$ is a LP-type problem.

\footnotetext{
${ }^{23}$ The other direction follows from the monotonicity property.

${ }^{24}$ Amenta [4] asserts that a "standart perturbation argument" can also be used.
} 


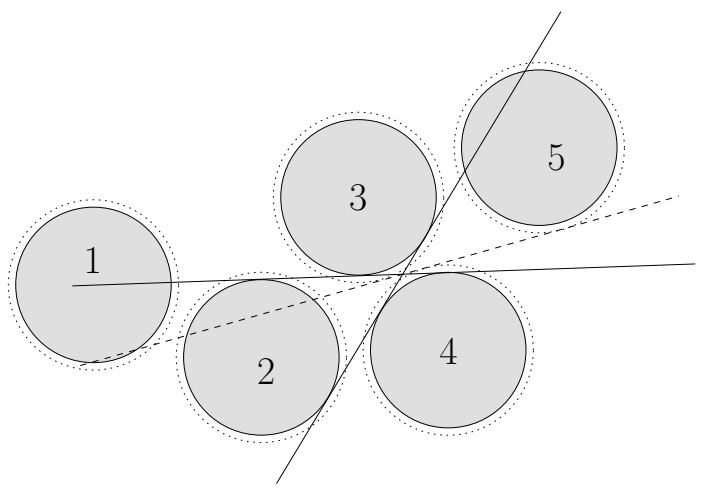

FIG. 9. $\phi$ may not fulfill the locality condition (with $F=\{2,3,4\}, G=F+5$ and $x=1$ ).

Proof. Let $F, G \subset \mathcal{C}$ and $x \in \mathcal{C}$. If $\phi(F)<N$ then $w(F+x)=w(F)$ if and only if $x$ intersects every transversal to $\phi(F) F$, so the monotonicity follows. If $F \subset G$ and $w(F)=w(G)$ then $\phi(F) F$ and $\phi(G) G$ have exactly the same set of transversals, and the locality follows.

To prove Theorem 6.1, it suffices to observe that the combinatorial dimension of $(\mathcal{C}, w)$ is bounded:

LEMMA 6.2. For all collections $\mathcal{C}$ of disjoint balls in $\mathbb{R}^{d}$ with radius disparity at most $\gamma$ the combinatorial dimension of $(\mathcal{C}, w)$ is $O\left(d^{2} \gamma^{\log \gamma}\right)$.

Proof. Let $F \subset \mathcal{C}$ be a basis and denote by $H(d, \gamma)$ the size of the largest family of disjoint balls in $\mathbb{R}^{d}$ with radius disparity at most $\gamma$ that has no transversal and is minimal for this property. Theorem 5.4 gives that:

$$
H(d, \gamma)=O\left(d^{2} \gamma^{\log \gamma}\right) \text {. }
$$

Define:

$$
\begin{gathered}
\rho=\max \{\phi(B) \mid B \subset F, B \neq F\}, \quad \text { and } \\
\mu=\max \{\nu(B) \mid B \subset F, B \neq F, \phi(B)=\phi(F)\} .
\end{gathered}
$$

If $\rho \neq \phi(F)$ then for any $\eta \in(\rho, \phi(F))$, the family $\eta F$ has no transversal but all its proper subsets do, and thus $|F| \leq H(n, \gamma)$. If $\rho=\phi(F)$ then let $B$ be a basis contained in $F$ such that $\phi(B)=\phi(F)$ and $\nu(B)=\mu$. By definition of $\mu$, for any proper subset $B^{\prime}$ of $B$ we have $\phi\left(B^{\prime}\right) \neq \phi(B)$ and so the previous argument yields that $B$ has size at most $H(d, \gamma)$. Each transversal to $\phi(F) B$ that is not a transversal to $\phi(F) F$ misses some ball $\phi(F) X$ with $X \in F \backslash B$. Thus, since $F$ is a basis, its size is at most $|B|+\mu-\nu(F)$. It follows from Theorem 4.1 that $\mu=O\left(\gamma^{\log \gamma}\right)$ and the statement follows. $\square$

REMARK 6.1. The same technique yields that the combinatorial dimension is at most $2 d-1$ for families of balls with a separation set of size 1 (using Theorem 5.2) and $4 d-1$ for families of disjoint unit balls (using Theorem 5.4). 
REMARK 6.2. If one defines

$$
\phi:\left\{\begin{aligned}
2^{\mathcal{C}} & \rightarrow & \Omega \\
F & \mapsto & \min (\{\rho \in[0,1] \mid \rho F \text { has an order-respecting transversal }\} \\
& & \bigcup\{N\})
\end{aligned}\right.
$$

then the problem $(\mathcal{C}, \phi)$ does not satisfy the locality assumption (see Figure 10). In this case, Theorem 3.2 ensures that the unique minimum property is satisfied.

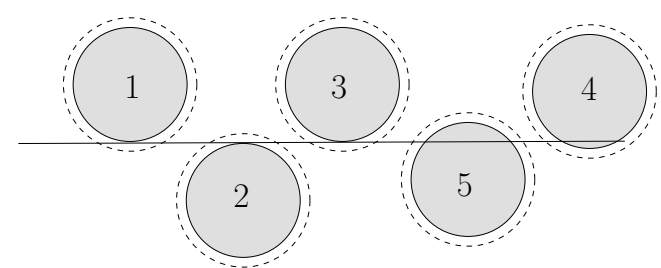

FIG. 10. For order-respecting transversals the locality condition is not satisfied (with $F=\{1,2,3\}, G=F+4$ and $x=5$ ).

7. Some open problems. To conclude this overview, we highlight a few of the many questions that remain open.

1. Geometric permutations. What is the asymptotic behavior of the maximum number of geometric permutations of $n$ disjoint convex sets in $\mathbb{R}^{d}$ ? The gap between the $\Omega\left(n^{d-1}\right)$ lower bound [73] and the $O\left(n^{2 d-2}\right)$ upper bound [78] was closed for disjoint balls [73] and fat objects [54] in $\mathbb{R}^{d}$ and narrowed for sets of bounded description complexity in three dimensions [55]. Also, what is the number of geometric permutations of $n \in\{4, \ldots, 9\}$ disjoint unit balls or to few disjoint balls with bounded radius disparity in dimension $d \geq 3$ ? A better grasp of these questions may be required to improve the current upper-bounds on the Helly number.

2. Hadwiger number of disjoint balls in $\mathbb{R}^{3}$. Are the pinning and Hadwiger numbers equal in 3 dimensions? In the plane, the argument used in Theorem 5.1 can be refined to prove that they are; intuitively, case analysis shows that if three objects pin an order-respecting transversal that does not intersect a fourth one, then three of the objects have no order-respecting transversal. Since this analysis exploits the fact that in two dimensions lines are also hyperplanes, it is not clear whether it generalizes to higher dimensions.

3. Pinning number of convex sets. Are there arbitrarily large minimal pinning configurations of convex sets in $\mathbb{R}^{d}$, or are the corresponding pinning numbers also bounded? Note that there are minimal pinning configurations of size six in $\mathbb{R}^{3}$ if the objects are not required to be strictly convex (see Figure 11).

Acknowledgements. The author is grateful to Boris Aronov, Ciprian Borcea, Otfried Cheong, Olivier Devillers, Hazel Everett, Andreas Holmsen and Sylvain Petitjean for helpful discussions. 


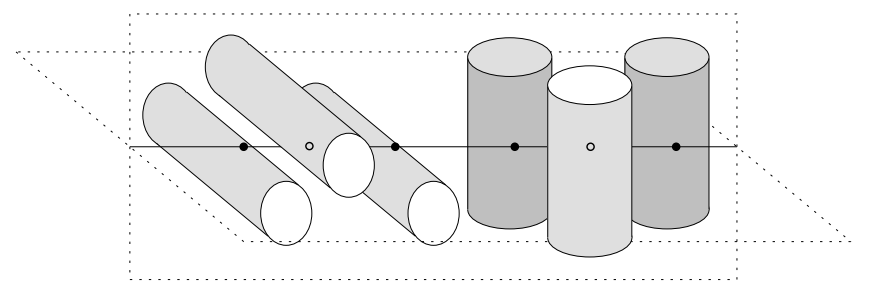

FIG. 11. A minimal pinning configuration consisting of 6 pieces of cylinder: the line is constrained to remain in two planes by triples of cylinders.

\section{REFERENCES}

[1] P. K. Agarwal, B. ARONov, AND M. ShariR, Line transversals of balls and smallest enclosing cylinders in three dimensions, Discrete \& Computational Geometry, 21 (1999), pp. 373-388.

[2] N. Alon AND G. KALAI, Bounding the piercing number, Discrete \& Computational Geometry, 13 (1995), pp. 245-256.

[3] G. Ambrus, A. Bezdek, And F. Fodor, A Helly-type transversal theorem for $n$ dimensional unit balls, Archiv der Mathematik, 86 (2006), pp. 470-480.

[4] N. AmEntA, Helly-type theorems and generalized linear programming, Discrete \& Computational Geometry, 12 (1994), pp. 241-261.

[5] - A new proof of an interesting Helly-type theorem, Discrete \& Computational Geometry, 15 (1996), pp. 423-427.

[6] B. ARONOV AND S. SMORODINSKy, On geometric permutations induced by lines transversal through a fixed point, Discrete \& Computational Geometry, 34 (2005), pp. 285-294.

[7] A. AsinowsKI, Common transversals and geometric permutations, master thesis, Technion IIT, Haifa, 1999.

[8] A. Asinowski, A. Holmsen, And M. Katchalski, The triples of geometric permutations for families of disjoint translates, Discrete Mathematics, 241 (2001), pp. 23-32.

[9] A. Asinowski, A. Holmsen, M. Katchalski, And H. TVerberg, Geometric permutations of large families of translates, in Discrete and Computational Geometry: The Goodman-Pollack Festschrift, B. Aronov, S. Basu, J. Pach, and M. Sharir, eds., vol. 25 of Algorithms and Combinatorics, Springer-Verlag, 2003, pp. 157-176.

[10] A. AsINOWSKI AND M. KATCHALSKI, Forbidden families of geometric permutations in $\mathbb{R}^{d}$, Discrete \& Computational Geometry, 34 (2005), pp. 1-10.

[11] - The maximal number of geometric permutations for $n$ disjoint translates of a convex set in $\mathbb{R}^{3}$ is $\omega(n)$, Discrete \& Computational Geometry, 35 (2006), pp. 473-480.

[12] D. AVIS, J.-M. ROBERT, AND R. WENGER, Lower bounds for line stabbing, Information processing letters, 33 (1989), pp. 59-62.

[13] D. Avis AND R. Wenger, Polyhedral line transversals in space, Discrete \& Computational Geometry, 3 (1988), pp. 257-265.

[14] M. BERn AND D. EPPSTEIn, Multivariate regression depth, Discrete \& Computational Geometry, 28 (2002), pp. 1-17.

[15] B. Bhattacharya, J. Czyzowicz, P. Egyed, G. Toussaint, I. Stojmenovic, and J. URRUTIA, Computing shortest transversals of sets, International Journal of Computational Geometry and Applications, 2 (1992), pp. 417-435.

[16] C. Borcea, X. Goaoc, And S. Petitjean, Line transversals to disjoint balls, Discrete \& Computational Geometry, 1-3 (2008), pp. 158-173.

[17] O. CheOng, X. GoAOC, AND A. Holmsen, Hadwiger and Helly-type theorems for disjoint unit spheres in $\mathbb{R}^{3}$, in Proc. 20th Ann. Symp. on Computational Geometry, 2005, pp. 1015.

[18] - Lower bounds for pinning lines by balls. Manuscript, 2008.

[19] O. Cheong, X. Goaoc, A. Holmsen, and S. Petitjean, Hadwiger and Helly-type theo- 
rems for disjoint unit spheres, Discrete \& Computational Geometry, 1-3 (2008), pp. 194212.

[20] O. CHEONG, X. GoAoc, AND H.-S. NA, Disjoint unit spheres admit at most two line transversals, in Proc. 11th Annu. European Sympos. Algorithms, vol. 2832 of Lecture Notes in Computer Science, 2003, pp. 127-135.

[21] _ Geometric permutations of disjoint unit spheres, Computational Geometry: Theory \& Applications, 30 (2005), pp. 253-270.

[22] F. Y. L. CHIN AND F. L. WANG, Efficient algorithm for transversal of disjoint convex polygons, Information processing letters, 83 (2002), pp. 141-144.

[23] L. DANZER, Über ein Problem aus der kombinatorischen Geometrie, Archiv der Mathematik, (1957).

[24] L. Danzer, B. Grünbaum, And V. Klee, Helly's theorem and its relatives, in Convexity, V. Klee, ed., Proc. of Symposia in Pure Math., Amer. Math. Soc., 1963, pp. 101-180.

[25] H. Debrunner, Helly type theorems derived from basic singular homology, American Mathematical Monthly, 77 (1970), pp. 375-380.

[26] F. DURAND, A multidisciplinary survey of visibility, in ACM SIGGRAPH Course Notes: Visibility, Problems, Techniques, and Applications, 2000.

[27] J. ECKHOFF, Helly, Radon and Caratheodory type theorems, in Handbook of Convex Geometry, J. E. Goodman and J. O'Rourke, eds., North Holland, 1993, pp. 389-448.

[28] H. EDELSBRUNNER AND M. SHARIR, The maximum number of ways to stab $n$ convex nonintersecting sets in the plane is $2 n-2$, Discrete \& Computational Geometry, 5 (1990), pp. 35-42.

[29] P. EgYed AND R. Wenger, Stabbing pairwise disjoint translates in linear time, in Proc. 5th Symposium on Computational Geometry, 1989, pp. 364-369.

[30] H. Everett, J.-M. Robert, AND M. VAN KREVELD, An optimal algorithm for the $(\leq k)$ levels, with applications to separation and transversal problems, in Proc. 9th Symposium on Computational Geometry, 1993, pp. 38-46.

[31] J. E. Goodman, When is a set of lines in space convex?, Notices of the AMS, 45 (1998), pp. 222-232.

[32] J. E. Goodman, A. Holmsen, R. Pollack, K. Ranestad, and F. Sottile, Cremona convexity, frame convexity, and a theorem of Santalo, Advances in Geometry, 6 (2006), pp. 301-322.

[33] J. E. Goodman And R. Pollack, Foundations of a theory of convexity on affine grassmann manifolds, Mathematika, 42 (1995), pp. 308-328.

[34] - Geometric transversal theory, in Encyclopaedia of Mathematics, Springer-Verlag, Heidelberg, Germany, 2002. http://eom. springer. de/g/g130050.htm.

[35] J. E. Goodman, R. Pollack, and R. Wenger, Geometric transversal theory, in New Trends in Discrete and Computational Geometry, J. Pach, ed., vol. 10 of Algorithms and Combinatorics, Springer-Verlag, Heidelberg, Germany, 1993, pp. 163-198.

[36] E. GREENSTEIN AND M. SHARIR, The space of line transversals to pairwise disjoint balls in $\mathbb{R}^{3}$. manuscript, 2005.

[37] B. GRÜnBAUM, On common transversals, Archiv der Mathematik, 9 (1958), pp. 465-469.

[38] _ Common transversals for families of sets, Journal of the London Mathematical Society, 35 (1960), pp. 408-416.

[39] B. Grünbaum and T. MotZkin, On components in some families of sets, Proceedings of the American Mathematical Society, 12 (1961), pp. 607-613.

[40] H. Hadwiger, Problem 107. Nieuw Archiv Wiskunde, (3)4:57, 1956; Solution. Wiskundige Opgaven, 20:27-29, 1957.

[41] _ U Über eibereiche mit gemeinsamer treffgeraden, Portugal Math., 6 (1957), pp. 23-29.

[42] E. HeLlY, Über Mengen konvexer Körper mit gemeinschaftlichen Punkten, Jahresbericht Deutsch. Math. Verein., 32 (1923), pp. 175-176.

[43] _ Über Systeme von abgeschlossenen Mengen mit gemeinschaftlichen Punkten, Monaths. Math. und Physik, 37 (1930), pp. 281-302.

[44] A. Holmsen, Recent progress on line transversals to families of translated ovals, in Computational Geometry - Twenty Years Later, J. E. Goodman, J. Pach, and R. Pollack, eds., AMS, 2008, pp. 283-298. 
[45] A. Holmsen, M. Katchalski, And T. Lewis, A Helly-type theorem for line transversals to disjoint unit balls, Discrete \& Computational Geometry, 29 (2003), pp. 595-602.

[46] A. Holmsen AND J. MATOUŠEK, No Helly theorem for stabbing translates by lines in $\mathbb{R}^{d}$, Discrete \& Computational Geometry, 31 (2004), pp. 405-410.

[47] Y. HuAnG, J. XU, AND D. Z. ChEn, Geometric permutations of high dimensional spheres, in Proc. 12th ACM-SIAM Sympos. Discrete Algorithms, 2001, pp. 244-245.

[48] - Geometric permutations of high dimensional spheres, Computational Geometry: Theory \& Applications, 29 (2004), pp. 47-60.

[49] J. JAROMCZYK AND M. KowALUK, Skewed projections with an application to line stabbing in $\mathbb{R}^{3}$, in Proc. 4th Conference on Computational Geometry, 1988, pp. 362-370.

[50] M. KatChALSKI, T. LEWIS, AND A. LiU, Geometric permutations and common transversals, Discrete \& Computational Geometry, 1 (1986), pp. 371-377.

[51] Geometric permutations of disjoint translates of convex sets, Discrete Mathematics, 65 (1987), pp. 249-259.

[52] — The different ways of stabbing disjoint convex sets, Discrete \& Computational Geometry, 7 (1992), pp. 197-206.

[53] M. KATChALSKI, S. SURI, AND Y. ZHOU, A constant bound for geometric permutations of disjoint unit balls, Discrete \& Computational Geometry, 29 (2003), pp. 161-173.

[54] M. J. KatZ AND K. R. VARADARAJAN, A tight bound on the number of geometric permutations of convex fat objects in $\mathbb{R}^{d}$, Discrete \& Computational Geometry, 26 (2001), pp. 543-548.

[55] V. KOLTUN AND M. SHARIR, The partition technique for overlays of enveloppes, SIAM Journal of Computing, 32 (2003), pp. 841-863.

[56] The Maple System. Waterloo Maple Software. http://www . maplesoft.com.

[57] J. MATOUŠEK, Lectures on Discrete Geometry, Springer-Verlag, 2002.

[58] J. MatousĚK, A Helly-type theorem for unions of convex sets, Discrete \& Computational Geometry, 18 (1997), pp. 1-12.

[59] N. MEGIDDO, On the complexity of some geometric problems in unbounded dimension, Journal of Symbolic Computation, 10 (1990), pp. 327-334.

[60] G. Megyesi AND F. Sottile, The envelope of lines meeting a fixed line and tangent to two spheres, Discrete \& Computational Geometry, 33 (2005), pp. 617-644. arXiv math.AG/0304346.

[61] J. Mitchell AND M. ShariR, New results on shortest paths in three dimensions, in Proc. 20th Symposium on Computational Geometry, 2004, pp. 124-133.

[62] J. O'ROURKE, An on-line algorithm for fitting straight lines between data ranges, Communications of the ACM, 24 (1981), pp. 574-579.

[63] J. PACH AND M. SharIR, Combinatorial Geometry with Algorithmic Applications - The Alcala Lectures. Alcala (Spain), August 31 - September 5, 2006.

[64] M. Pellegrini, Ray shooting and lines in space, in Handbook of Discrete \& Computational Geometry, J. E. Goodman and J. O’Rourke, eds., CRC Press LLC, 2004, ch. 37, pp. 839856.

[65] H. Pottmann and J. Wallner, Computational Line Geometry, Springer, 2001.

[66] C. V. Robinson, Spherical theorems of Helly type and congruence indices of spherical caps, American Journal of Mathematics, 64 (1942), pp. 260-272.

[67] P. Rousseeuw and M. Hubert, Regression depth, J. Amer. Stat. Assoc., 94 (1999), pp. 388 402.

[68] L. SAntaló, Un theorema sobre conjuntos de paralelepipedos de aristas paralelas, Publ. Inst. Mat. Univ. Nat. Litoral, 2 (1940), pp. 49-60.

[69] R. SEIDEL, Small-dimensional linear programming and convex hulls made easy, Discrete \& Computational Geometry, 6 (1991), pp. 423-434.

[70] M. SHARIR AND S. SMORODINSKY, On neighbors in geometric permutations, Discrete Mathematics, 268 (2003), pp. 327-335.

[71] M. SHARIR AND E. WELZL, A combinatorial bound for linear programming and related problems, in Proc. 9th Sympos. on Theo. Aspects of Comp. Science, 1992, pp. 569-579.

[72] S. SMORODINSKY, Geometric permutations and common transversals, master thesis, Tel Aviv University, Haifa, 1998. 
[73] S. SMORODINSKY, J. S. B. MitCHELl, AND M. SHARIR, Sharp bounds on geometric permutations for pairwise disjoint balls in $\mathbb{R}^{d}$, Discrete \& Computational Geometry, 23 (2000), pp. $247-259$.

[74] F. SotTILE AND T. THeOBALD, Line problems in nonlinear computational geometry, in Computational Geometry - Twenty Years Later, J. E. Goodman, J. Pach, and R. Pollack, eds., AMS, 2008, pp. 411-432.

[75] H. TVerberg, Proof of Grünbaum's conjecture on common transversals for translates, Discrete \& Computational Geometry, 4 (1989), pp. 191-203.

[76] P. VINCENSINI, Figures convexes et variétés linéaires de l'espace euclidien à $n$ dimensions, Bull. Sci. Math., 59 (1935), pp. 163-174.

[77] R. WENGER, A generalization of hadwiger's transversal theorem to intersecting sets, Discrete \& Computational Geometry, 5 (1990), pp. 383-388.

[78] — Upper bounds on geometric permutations for convex sets, Discrete \& Computational Geometry, 5 (1990), pp. 27-33.

[79] _ Helly-type theorems and geometric transversals, in Handbook of Discrete \& Computational Geometry, J. E. Goodman and J. O'Rourke, eds., CRC Press LLC, Boca Raton, FL, 2nd ed., 2004, ch. 4, pp. 73-96.

[80] Y. ZHOU AND S. SURI, Shape sensitive geometric permutations, in Proc. 12th ACM-SIAM Sympos. Discrete Algorithms, 2001, pp. 234-243.

[81] - Geometric permutations of balls with bounded size disparity, Computational Geometry: Theory \& Applications, 26 (2003), pp. 3-20. 